\title{
First observations of the second solar spectrum with spatial resolution at the Lunette Jean Rösch ${ }^{\star}$
}

\author{
J.-M. Malherbe ${ }^{1}$, J. Moity ${ }^{1}$, J. Arnaud ${ }^{2}$, and Th. Roudier ${ }^{3}$ \\ 1 LESIA, Observatoire de Paris, Section de Meudon, 92195 Meudon Cedex, France \\ e-mail: jean-marie.malherbe@obspm.fr \\ 2 Laboratoire d'Astrophysique, Observatoire Midi Pyrénées, 14 avenue Edouard Belin, 31400 Toulouse, France \\ 3 Laboratoire d'Astrophysique, Observatoire Midi Pyrénées, 57 avenue d'Azereix, PB 826, 65008 Tarbes Cedex, France
}

Received 26 June 2006 / Accepted 28 September 2006

ABSTRACT

\begin{abstract}
Context. A new polarimeter has been installed at the focus of the $50 \mathrm{~cm}$ refractor of the Lunette Jean Rösch (LJR), previously known as Turret Dome, Pic du Midi, France, for spectroscopic observations of weak solar magnetic fields. Fields can be derived through the Hanle effect from the depolarization of the second solar spectrum (i.e. the linearly polarized spectrum at the limb).

Aims. We present the first observations with spatial resolution based on the new device performed with the large $8 \mathrm{~m}$ Echelle spectrograph, or recorded in imagery mode through narrow band filters. The observations started in April 2004, especially in the blue part of the spectrum where our instrumentation has a particularly good efficiency. The capabilities and the characteristics of the new instrument are briefly described. We observed several lines of the second solar spectrum with the slit of the spectrograph orthogonal to the limb to study the polarization as a function of limb distance (which is related to altitude in the atmosphere), and several spectral windows in imagery to determine the average continuum polarization.

Methods. The polarimeter uses Nematic Liquid Crystal (NLC) technology at the primary focus of the refractor, in spectroscopic or imagery mode.

Results. A continuous polarization profile through the limb is presented for the photospheric SrI $460.7 \mathrm{~nm}$ line, the low chromospheric BaII $455.4 \mathrm{~nm}$ line, and the CaI $422.7 \mathrm{~nm}$ line within a distance of 120", together with measurements of the mean continuum polarization obtained in imagery mode. Preliminary results of the polarization of the SrI $460.7 \mathrm{~nm}$ line are also shown at $40^{\prime \prime}$ from the limb, as a function of the brightness of structures visible in the continuum (granulation). They reveal a tendency for the polarization to be weaker in dark features (intergranules) than in bright ones (granules), suggesting a stronger magnetic field in intergranular lanes. As example the enigmatic and weak polarization signal in the core of the $\mathrm{NaD} 1589.6 \mathrm{~nm}$ line is presented.

Conclusions. Some aspects of the spatial variation of the polarization with respect to the granulation pattern require further investigation at higher spatial resolution.
\end{abstract}

Key words. polarization - instrumentation: polarimeters - instrumentation: spectrographs - Sun: photosphere -

Sun: chromosphere - Sun: magnetic fields

\section{Introduction}

The second solar spectrum is the linearly polarized spectrum observed on the limb of the sun. It is as rich as the ordinary intensity spectrum, but the two spectra are very different. The second solar spectrum is generated by coherent scattering due to the anisotropy of the radiation field, both with and without magnetic fields. The polarization rate is generally small (typical $1 \%$ or less) and decreases in the presence of magnetic fields. This well known effect is called Hanle depolarization and is generally observed in Stokes $Q / I$, which characterizes the linear polarization parallel to the limb. But another aspect of the Hanle effect (rotation of the plane of polarization) may also induce signals in Stokes $U / I$. The first spectral survey of the second solar spectrum was performed at the Kitt Peak Observatory in Stokes $Q / I$ by Stenflo et al. $(1983 a, b)$ from the Ultra Violet to the near Infra Red. It was then followed by a much more sensitive survey by Gandorfer (2000, 2002) using the ZIMPOL polarimeter. The second solar spectrum requires polarization free telescopes to be best recorded; for optical designs that introduce instrumental

* Appendices A-C are only available in elctronic form at http://www . aanda.org polarization (such as coelostats or Coudé telescopes), specific calibrations or epochs of observation (such as the equinoxes) may be necessary. Telescopes or refractors with axial symmetry such as THEMIS or the Pic du Midi LJR are well suited for this.

Observations of the second solar spectrum opened a new field of investigation and have led to many applications: in atomic physics, new polarization phenomena have been discovered, involving rare atomic elements, molecules and unexplored processes of quantum mechanics, such as interference, optical pumping or hyperfine structure; in solar physics, new diagnostics of spatially unresolved turbulent and weak magnetic fields have been proposed, in a complementary way to the Zeeman effect for stronger and resolved fields. For a comprehensive overview, see Stenflo (2003a,b, 2004, 2006).

The second solar spectrum has many polarized lines, as shown by atlases, but many transitions depolarize the continuum. Line depressions below the continuum polarization level look like absorption lines of the intensity spectrum, while lines more polarized than the continuum look like emission lines. Stenflo (2004) noticed from observations made at two different periods (1995, close to the minimum activity, and 2000, near maximum) 
that the proportion of absorption-like and emission-like transitions varies, which suggests the influence of hidden (weak and unresolved) magnetic fields, through the Hanle depolarization mechanism (less polarization at the solar maximum in the presence of stronger magnetics fields). Hence, the analysis of long term variations of the second solar spectrum could be a powerful tool to follow the magnetism along the solar cycle.

We describe the new Nematic Liquid Crystal (NLC) polarimeter operating at the Pic du Midi LJR since April 2004, following a previous version (installed in 2003) described by Malherbe et al. (2004), which used another technology (Ferroelectric Liquid Crystal, FLC). We present the first observations obtained with this new instrument in various lines, with spatial resolution, in spectroscopic mode, or in imagery mode through narrow band filters, and compare our results to ones in the literature.

\section{Experiment setup}

The experiment setup is described in detail in the appendices or on line at the Web page http://www.lesia.obspm.fr/ $\sim$ malherbe/papers/index.html. We give here a short summary.

The Pic du Midi LJR is a $50 \mathrm{~cm}$ aperture refractor $(f=6.5 \mathrm{~m})$ supported by an equatorial mount. The beam has axial symmetry along the optical axis. Polarization analysis is achieved before transmission to the spectrograph by a flat mirror at $45^{\circ}$ (Fig. 1), therefore instrumental polarization is minimized. The spatial resolving power is $0.3^{\prime \prime}$. The primary image is magnified 10 times at the secondary focus $\mathrm{F} 2(f=65 \mathrm{~m})$ where the slit of the spectrograph is located. We used a new prototype of a liquid crystal polarimeter between focus F1 and F2. It was used for the first time in April 2004. The polarimeter has the following elements (Fig. 1):

- two variable retarders (retardance continuously adjustable between 0 and $700 \mathrm{~nm}$ ) which combine a single Nematic Liquid Crystal cell with a static birefringent element;

- a precision dichroic linear polarizer located at the exit of the system, on the optical axis, before the reflecting mirror to the spectrograph.

The signal $\mathrm{S}$ provided by the polarimeter is given by

$S=\frac{1}{2}(I+Q \cos \delta 2+\sin \delta 2(U \sin \delta 1-V \cos \delta 1))$

where $I, Q, U$ and $V$ are the Stokes parameters and $\delta 1, \delta 2$ are the retardance of the two NLC.

We used for this work a simplified version of the polarimeter, running only with one retarder $(\delta 1=0)$ so that the output signal becomes $S=\frac{1}{2}(I+Q \cos \delta-V \sin \delta)$. Stokes parameters $I \pm V$ are obtained sequentially with $\delta=(\pi / 2,3 \pi / 2)$. This property was used by Roudier et al. (2006) for high resolution Zeeman magnetometry on the disk. In the present paper, we are interested only in $I \pm Q$ which were derived from this formula with $\delta=(0, \pi)$.

Since measurements of $I \pm P$ (where $P$ is any Stokes parameter) are not simultaneous, our polarimeter can operate with a good efficiency in two particular domains:

- observations of the line of sight magnetic fields on the disk, with the 2D imaging spectroscopy device provided by the Multichannel Subtractive Double Pass (MSDP), as described by Malherbe et al. (2004) and Mein (2002);

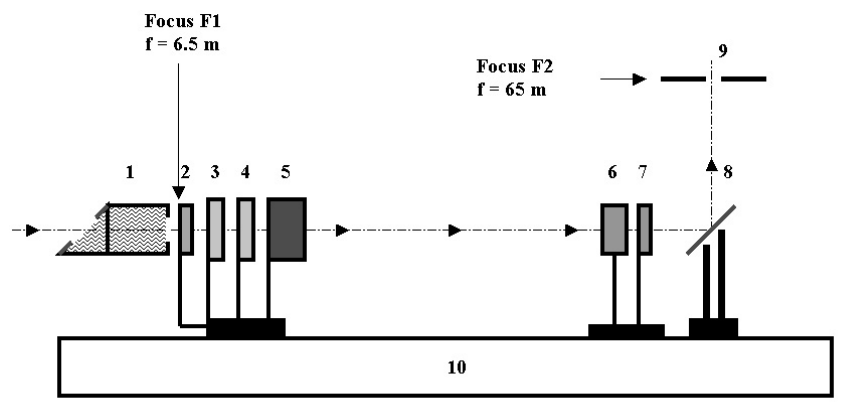

6: interference filter

water cooling device (field stop) 2: UV/IR filter $380-700 \mathrm{~nm}$ 3: variable retarder 1

4: variable retarder 2

5: magnification lens $(\mathrm{f}=60 \mathrm{~mm})$

7: precision dichroïc

8: flat mirror

9: spectrograph entrance slit 10: optical rail

Fig. 1. The optical setup between the primary focus F1 and the secondary focus F2 in spectroscopic mode (the $50 \mathrm{~cm}$ refractor is located on the optical axis at left).

- observations of the second solar spectrum; since the polarization degrees are small, the signal has to be derived from the statistical analysis of hundreds or thousands of spectra obtained sequentially, in order to improve the signal to noise ratio. Since the spectrograph has a good transmission in the blue part of the spectrum, our observations are mainly focused in this range of the second solar spectrum. We started observations with two scientific goals:

1. measurement of weak polarizations $(0.01 \%)$ at moderate spatial resolution: when the seeing conditions are not excellent, we use a $0.6^{\prime \prime} \times 140^{\prime \prime}$ slit and accumulate a large number of spectra to achieve the best polarimetric sensitivity as possible; data can be averaged along the slit to reduce the noise;

2. measurement of stronger polarizations $(0.1 \%$ to $1 \%)$ at higher spatial resolution: when the seeing conditions are fairly good, we use a thin slit of $0.3^{\prime \prime} \times 140^{\prime \prime}$ to investigate the polarization in the structures of the sun.

We used the $8 \mathrm{~m}$ Littrow Echelle spectrograph (Mouradian et al. 1980). The interference order is isolated by $10 \mathrm{~nm}$ bandpass filters (order 10 at $590 \mathrm{~nm}$ for $\mathrm{NaD1}, 13$ at $455 \mathrm{~nm}$ for SrI and BaII, 14 at $420 \mathrm{~nm}$ for $\mathrm{CaI}$ ). For the previous orders, the dispersion lies respectively around $55 \mathrm{~mm}, 72 \mathrm{~mm}$ and $77 \mathrm{~mm}$ per nanometer. The spectrum obtained at the focus of the spectrograph $(60 \mathrm{~mm} \times 90 \mathrm{~mm})$ is reduced about 10 times on the CCD camera, so that the spatial pixel size on the detector is $0.2^{\prime \prime}$ along the slit direction and the spectral pixel size is $1.1 \mathrm{pm}$ (in the blue part of the spectrum). The exposure time was typically $50 \mathrm{~ms}$ during our runs around $450 \mathrm{~nm}$ at $\mu=0.15$ (limb distance of $\left.10^{\prime \prime}\right)$ with the $0.6^{\prime \prime} \times 140^{\prime \prime}$ slit. Since a pair of images $I \pm Q$ has a photometric precision of about $10^{-2}$, one hour of observation at the limb at $450 \mathrm{~nm}$ allows to reach a precision of roughly $10^{-4}$. It is even possible to measure weaker polarization signals by integrating in the solar direction along the slit. In the case of total integration, a single pair of images $I \pm Q$ will deliver a precision better than $10^{-3}$, and after one hour of observations, a ratio better than $10^{-5}$ can be achieved.

Flat field observations are made around disk center in the quiet sun where linear polarization signals should vanish. It is essential to precisely determine the transmission of the instrument for the two states of polarization. The zero level is a function of wavelength in the spectral range (typical $Q / I$ slope of about $5 \times$ $10^{-4}$ per $\mathrm{nm}$ which is corrected in data processing). 
In the following, full sets of data consisting of thousands of $I \pm Q$ images of the same region at the limb, plus a series of flat field images taken at disk center in both polarizations and terminated by dark current images, are analysed.

\section{Stability of the polarization and effect of cirrus}

The stability of polarization measurements may be affected by several effects, such as solar, but also seeing and instrumental variations. It was noticed in the past that the image quality deteriorates when the main objective holder is heated by the sun. For that reason, P. Mehltretter suggested to protect the holder by a reflective ring with a clear aperture corresponding to the diameter of the lens. We discovered recently that instrumental depolarization (up to $-25 \%$ ) occurs without the ring two hours before and after the meridian. In order to check the stabilizing effect of the ring, we observed continuously, on May 10, 2005, the linear polarization of $\mathrm{SrI} 460.7 \mathrm{~nm}$ at the South pole with the slit of the spectrograph $\left(0.6^{\prime \prime} \times 140^{\prime \prime}\right)$ parallel to the solar limb, at about $10^{\prime \prime}(\mu=0.15)$. Results averaged along the slit are displayed in Fig. 2. While the intensity curve shows the variation of solar irradiance during observations, the polarization rate $Q / I$ measured in the line core lies around $0.8 \%$ to $0.9 \%$ with only small fluctuations $( \pm 0.05 \%)$, which are mainly due to variations of the limb distance which can change within a few arcsec between each test, as we do not have a precise guiding system. Thus, if some instrumental depolarization remains, it is not easily detectable.

During the numerous test runs, we checked the effects of cirrus, which are thin clouds of ice at high altitude in the troposphere (6000-10000 m), and other cirrus type clouds such as cirrocumulus, which are denser. The depolarizing effect of cirrus is well known and has been studied by atmospheric researchers (see for instance Chen et al. 2002) in the following way: they measure with a telescope the depolarization of the back- scattered light coming from a linearly polarized laser beam (Lidar depolarization). While the backscatter signal of linearly polarized laser light by spherical particles (cloud droplets) remains totally polarized, it is not the case when particles are non- spherical (such as ice crystals, snow or dust particles, aerosols). The classification of particle shapes is an application of Lidar depolarization in ice clouds (Noel et al. 2002, 2004). Here we checked related phenomena by measuring the depolarization of the solar SrI $460.7 \mathrm{~nm}$ and BaII $455.4 \mathrm{~nm}$ (Fig. 3) lines through cirrus or cirrocumulus of various optical thickness (density) along the line of sight. This quantity was easily determined using observations made a few minutes before or after their passage (it is defined by the relation $d=\log _{10}\left(I_{0} / I\right)$, where $I_{0}$ and $I$ are respectively the intensity in the absence or presence of clouds). The two figures show a strong depolarizing effect on the linear polarization of solar lines (up to a factor of 2 for dense cirrus). The depolarization mechanism is unclear: it may be due to the physical nature of the clouds, but an easier explanation can be proposed. Our experiment differs from Lidar measurements because the sun is an extended source, mostly unpolarized. When observing through clouds, an amount of scattered light which is not polarized could be included, because large amounts of such light come from the solar disk. Thus observing close to the limb, partially polarized light (generated by scattering effects in the solar atmosphere at the limb) could be mixed with unpolarized light (generated by scattering effects in the clouds of unpolarized light of the disk). In that case, the net result should be a decrease of the polarization signal measured with a clear sky. We

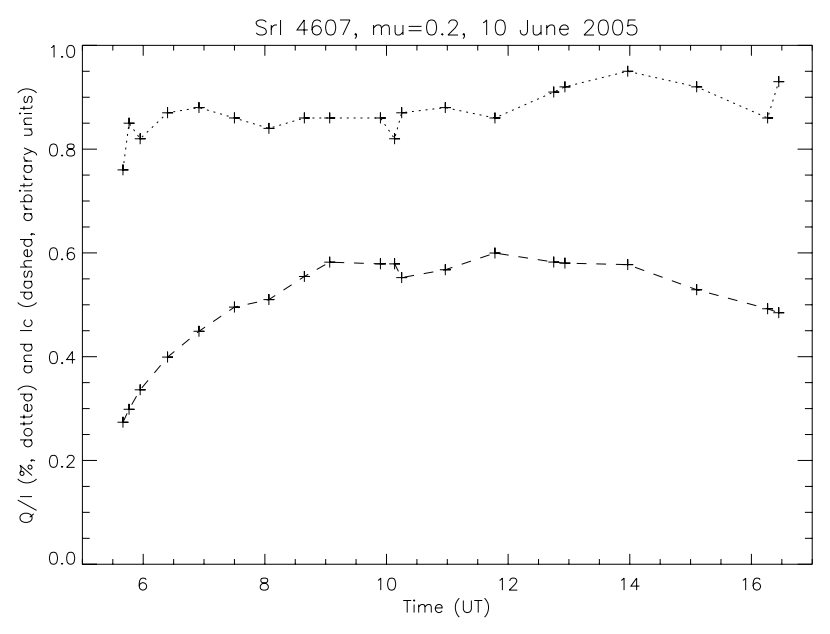

Fig. 2. Stability of the SrI $460.7 \mathrm{~nm}$ linear polarization. Dotted line: polarization rate $Q / I$ as a function of time measured at $\mu=0.15\left(10^{\prime \prime}\right.$ from the limb); dashed line: intensity $I$ (arbitrary units).

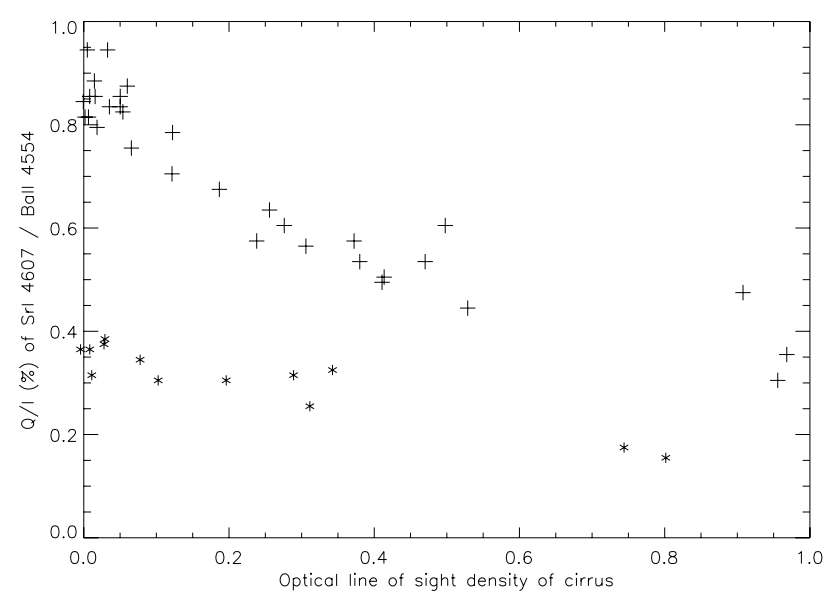

Fig. 3. Depolarization of the SrI $460.7 \mathrm{~nm}$ line (crosses) and the BaII $455.4 \mathrm{~nm}$ line (stars) by atmospheric cirrus as a function of cloud optical density for $\mu=0.15$.

conclude that clouds should be absolutely avoided during solar polarimetry experiments.

\section{Observations of the Srl $460.7 \mathrm{~nm}$ line}

On May 15, 2004, we observed the linear polarization of the SrI $460.7 \mathrm{~nm}$ line at the South pole with the slit of the spectrograph $\left(0.6^{\prime \prime} \times 140^{\prime \prime}\right)$ parallel to the limb, at various distances. For each distance $\left(10^{\prime \prime}, 20^{\prime \prime}, 40^{\prime \prime}, 80^{\prime \prime}\right.$ and $160^{\prime \prime}$ corresponding to $\mu=0.15,0.2,0.3,0.4,0.55)$, we obtained in a few minutes short series of 400 pairs of spectra $I \pm Q$, which were integrated along the solar direction to achieve a sensitivity smaller than $10^{-4}$ in the line core. The corresponding polarization rates were found respectively to be $1.0 \%, 0.8 \%, 0.6 \%, 0.4 \%$, and $0.2 \%$, as shown in Fig. 4. We estimate the precision of the limb distance at $\pm 1^{\prime \prime}$, due to seeing fluctuations and some mechanical instability of the refractor. The zero polarization level is fixed by the flat field at disk center.

On May 14, 2004, we also observed at the West limb with the slit of the spectrograph perpendicular to the limb. With this orientation of the slit, it is not possible to observe at the poles, because we cannot rotate the image of the sun at the focus of the refractor. We checked for the absence of active regions. A long 


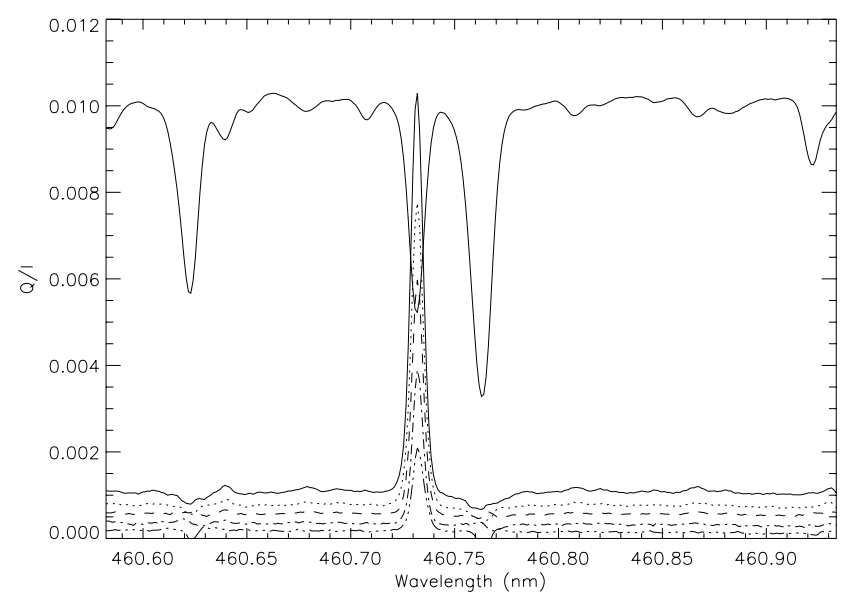

Fig. 4. $Q / I$ for the SrI $460.7 \mathrm{~nm}$ line at various distances of the limb $\left(10^{\prime \prime}, 20^{\prime \prime}, 40^{\prime \prime}, 80^{\prime \prime}\right.$ and $\left.160^{\prime \prime}\right)$ as a function of wavelength in $\mathrm{nm}$ (pixel size of $1.1 \mathrm{pm}$ ). The slit is parallel to the limb and data are averaged along the slit. The mean intensity is superimposed (thick line).

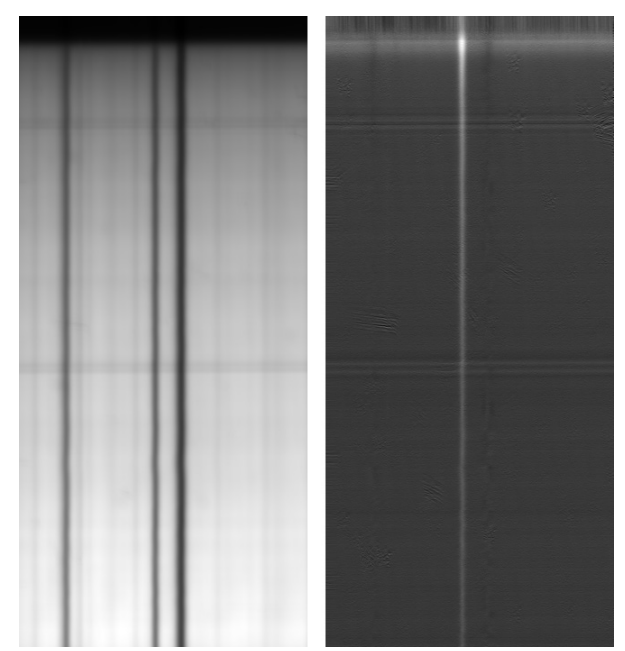

Fig. 5. I (left) and $Q / I$ (right) images of SrI $460.7 \mathrm{~nm}$. Abscissa: the wavelength (bandwidth of $0.35 \mathrm{~nm}$, pixel size of $1.1 \mathrm{pm}$ ); ordinates: the solar direction $x$ along the slit (field of view 140", pixel size $0.2^{\prime \prime}$ ).

sequence of 6400 pairs of images $I \pm Q$ was obtained over one hour, to reach a precision of $2 \times 10^{-4}$ in the line core for each pixel along the slit, and better in the continuum. Fluctuations of the limb positions were tracked and corrected by the software. Figure 5 shows the map $Q / I(\lambda, x)$ (where $\lambda$ is the wavelength and $x$ the spatial coordinate) obtained after the processing of the 12800 spectra. The limb is at the top of the figure. Polarization fringes are not visible because the polarization signal is high and increases towards the limb, both in the continuum and in the line core.

From Fig. 5, we derived the polarization profile in the line core and in the continuum as a function of limb distance, as presented in Fig. 6, where the spatial pixel was brought from $0.2^{\prime \prime}$ to $1.0^{\prime \prime}$ in order to improve the signal to noise ratio roughly to $10^{4}$. The polarization rate at the limb was found in the line core to be $1.8 \%$ (respectively $0.5 \%$ in the continuum). The error bar due to image motion, such as intensity fluctuations $\Delta I / I$ which correspond to seeing induced cross talk, increases dramatically from a few $0.01 \%$ to $0.2 \%$ at the limb where intensity vanishes. We suspect from Fig. 6 an offset of the zero polarization level of about $0.05 \%$ (visible on the continuum), which means

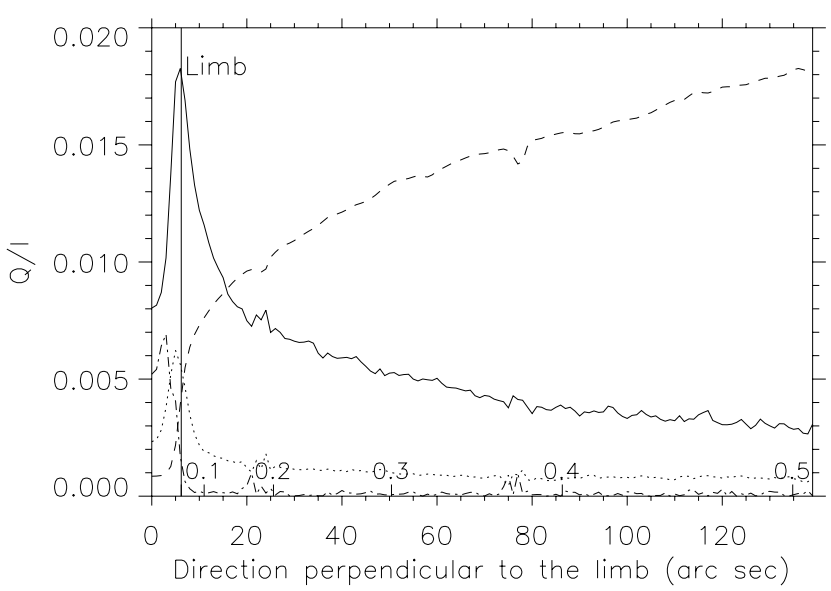

Fig. 6. $Q / I$ variations in the $\mathrm{SrI} 460.7 \mathrm{~nm}$ line centre (solid line) and in the continuum at $460.84 \mathrm{~nm}$ (dotted line) as a function of limb distance in $\operatorname{arcsec}$ (values of $\mu$ are indicated above $x$ axis). The signal to noise ratio is better for the continuum than for the line center, because we have integrated along 20 pixels in wavelength (bandwidth of $0.02 \mathrm{~nm}$ ). Defaults at abscissa 20 and 75 are due to transversalium (dust or defaults along the slit of the spectrograph). The dashed line is the intensity profile (arbitrary units), the limb is defined by the inflexion point. The dashed dotted line represents the error bar of $Q / I$ which is of the order of $10^{-4}$ except near the limb where image motion limits the accuracy to $10^{-3}$.

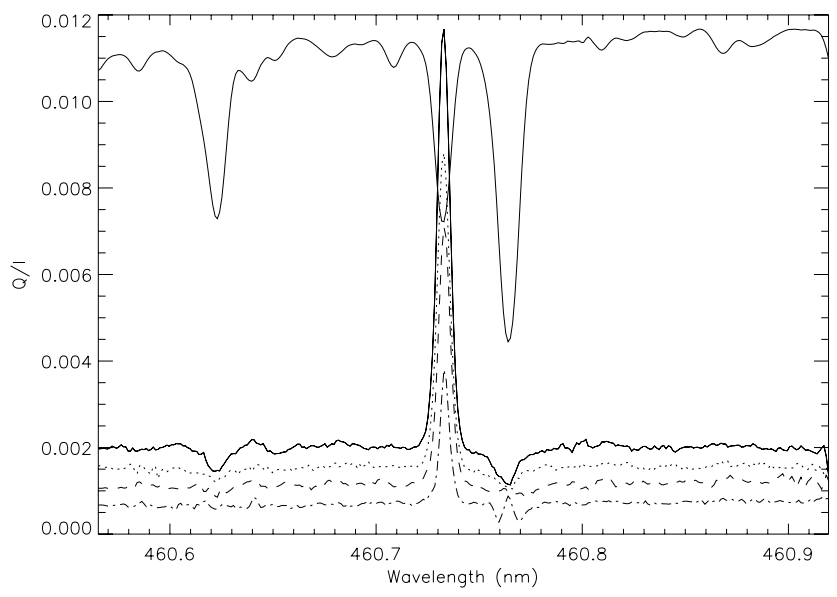

Fig. 7. $Q / I$ for the $\mathrm{SrI} 460.7 \mathrm{~nm}$ line at various distances of the limb $\left(5^{\prime \prime}, 10^{\prime \prime}, 20^{\prime \prime}, 80^{\prime \prime}\right)$ as a function of wavelength in $\mathrm{nm}$ (pixel size of $1.1 \mathrm{pm})$. The mean intensity curve is superimposed (thick line). The slit is orthogonal to the limb and data are averaged over $1^{\prime \prime}$.

that the zero established by the flat field has slightly shifted in time during the observing run. Up to now, determinations of $Q / I$ have been obtained mainly for some discrete distances of the limb (Stenflo et al. 1997; Faurobert et al. 2001; Bommier et al. 2005). Faurobert et al. (2001) obtained results that are consistent with the presence of a weak turbulent magnetic field between 20 and $30 \mathrm{G}$, while Bommier et al. (2005) deduced $46 \mathrm{G}$ from their observations. Sanchez Almeida (2005) showed that SrI $460.7 \mathrm{~nm}$ depolarization measurements indicate that $40 \%$ only of the quiet sun could be filled by magnetic fields weaker than $60 \mathrm{G}$, the remaining part being above the quiet sun. The spatial variations of the polarization signal shown in Fig. 6 over $120^{\prime \prime}$ were interpreted by Derouich et al. (2006), who determined vertical variations of the turbulent magnetic field in the sun's photosphere.

From Fig. 5, we also derived profiles of $Q / I(\lambda)$ (see Fig. 7) for various distances $\left(5^{\prime \prime}, 10^{\prime \prime}, 20^{\prime \prime}\right.$ and $80^{\prime \prime}$ corresponding to 
$\mu=0.1,0.15,0.2$ and 0.4$)$, which corroborate the results of Fig. 4 (except for the continuum). Our results at $5^{\prime \prime}$ from the limb $(Q / I=1.2 \%)$ are in good agreement with previous measurements. Gandorfer (2002) found a polarization peak of $1.2 \%$ at line center from observations made in 2001. Bommier \& Molodij (2002) obtained 1.15\%, and Faurobert et al. (2001) $0.95 \%$ from observations both made with THEMIS in 2000. Stenflo et al. (1997) reported 1.5\%. Other observations have been compiled by Trujillo Bueno et al. (2004). But all authors observed near the poles, so that the comparison with us is not straightforward (West limb), with different instruments and methods. We could expect the polarization rate to vary with the solar cycle; it could be lower near the maximum of the cycle, because the magnetic fields are stronger. But we think that the heterogeneity of measurements does not allow anyone to conclude firmly about variations along the 11 years cycle. Observing with the slit orthogonal to the limb has the advantage of allowing a precise determination $\left(1^{\prime \prime}\right)$ of the distance to the limb. We therefore suggest systematic observations along the solar cycle with the same setup, to study the temporal variation of the polarization of $\mathrm{SrI} 460.7 \mathrm{~nm}$ in quiet regions.

We investigated also the spatial variation of the polarization of the SrI $460.7 \mathrm{~nm}$ line at constant limb distance. According to Trujillo Bueno et al. (2004), the turbulent magnetic field in the granular regions could be much weaker than what is required to explain the depolarization in the SrI line. Hence, the intergranular regions could exhibit relatively strong fields capable of producing most of the SrI depolarization. For that reason, it is important to observe at constant limb distance with good spatial resolution in order to distinguish bright and dark features, such as granules or intergranules. Such observations require rare seeing conditions, with a thin slit $\left(0.3^{\prime \prime}\right)$. But, as the contrast decreases towards the limb, and as the perspective effect increases, it is not possible to observe as close to the limb as is usually done. We started this program on September 17, 2004, at medium resolution with a slit of $0.6^{\prime \prime}$ at $40^{\prime \prime}$ from the limb $(\mu=0.3)$, with a short exposure time $(50 \mathrm{~ms})$. We decided to class the large amount (300000) of observed spectra $I \pm Q(\lambda)$ as a function of the continuum intensity as seen in the vicinity of the SrI line core, the darkest regions corresponding to intergranular lanes and the brightest to the granules. As contrasts remain low, even at this limb distance, the data processing has to treat very carefully intensity fluctuations that may arise from the sky and atmospheric transmission variations. For this purpose, a light curve was drawn and used to correct each spectrum of the sequence in order to keep the same photometry. Individual spectra were classified into 20 intervals in increasing order of continuum intensity, then summed to improve the signal to noise ratio. Mean Stokes $I(\lambda)$ and $Q(\lambda)$ were computed, and the linear polarization rate $Q / I(\lambda)$ was derived for each interval of continuum intensity. The full range of available intensities varies from 0.94 to 1.06 times the average intensity, so that the amplitude of each interval corresponds to 0.006 times the mean continuum intensity. Inside each interval, the number of $I+Q$ and $I-Q$ profiles was kept identical in order to combine consecutive pairs of polarizations, but it changes from one interval to the next (as shown by Fig. 8, histogram of intensities). The polarization ratio $Q / I$ in the line core increases with the continuum intensity (Fig. 8), suggesting that the depolarization due to the Hanle effect could be higher in dark regions (intergranules) than in bright regions (granules), which could be the signature of higher magnetic fields in dark regions (intergranules). At our knowledge, this observation is the first one, so that this preliminary result needs to be confirmed by new observations at higher

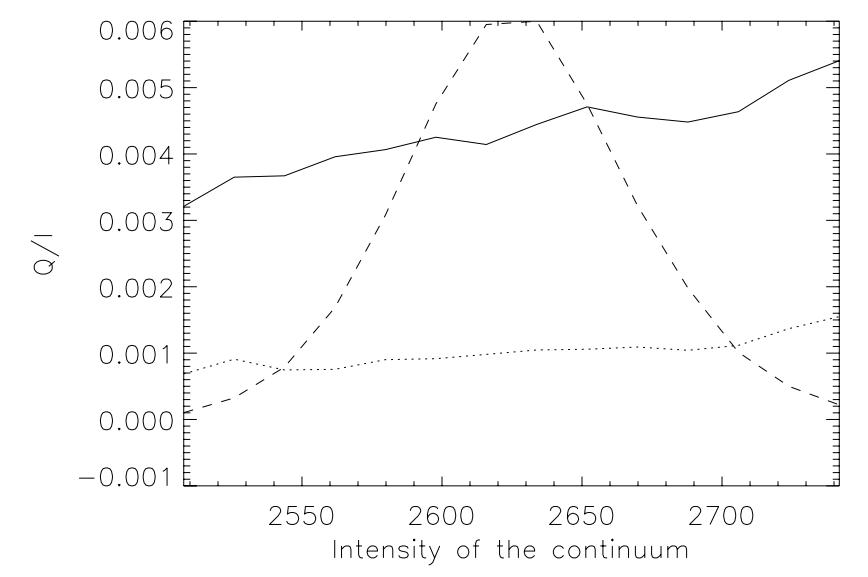

Fig. 8. Polarization in the SrI $460.7 \mathrm{~nm}$ line as a function of the continuum intensity (arbitrary units). The dashed line is the histogram of intensities and represents the number of spectra of each interval (the peak corresponds to 30000 spectra). The solid line is the $Q / I$ ratio in the line core, while the dotted line is the $Q / I$ ratio in the continuum. Values on the abscissa lie in the range 0.955 to 1.045 times the mean continuum value.

spatial resolution, with a thinner slit, and at various distances from the limb.

\section{Observations of the Ball $455.4 \mathrm{~nm}$ line}

Observations of the BaII $455.4 \mathrm{~nm}$ line were performed with the slit orthogonal to the limb on May 15, 2004. This line is of great interest because the core is formed in the low chromosphere. Observational diagnostics of the center to limb variation of the polarization may be used to investigate the weak and turbulent magnetic fields in the low chromosphere. Observations were made at the West limb, and during one hour, 8000 pairs of images $I \pm Q$ were recorded, in order to reach a precision of $2 \times$ $10^{-4}$ in the line core for each pixel along the slit, and better in the continuum. Figure 9 shows the map $Q / I(\lambda, x)$ obtained after the processing of the 16000 spectra which were coaligned both in the spatial and the spectral directions. The limb is at the top of the figure. A strong polarization in the low chromosphere can be noticed in the line core, above the solar limb defined by the continuum.

From Fig. 9, we derived the polarization profile in the line core and in the continuum as a function of limb distance, as presented in Fig. 10, where the pixel size is magnified to $1.0^{\prime \prime}$ in order to improve the signal to noise ratio roughly to $10^{4}$. The polarization rate in the line core at the limb was found to be $1.1 \%$ (respectively $0.5 \%$ in the continuum). The error bar due to image motion, such as intensity fluctuations $\Delta I / I$, increases drastically from a few $0.01 \%$ to $0.2 \%$ near the limb (where intensity vanishes). The polarization peaks of the line core and continuum are shifted by $2^{\prime \prime}$ to $3^{\prime \prime}$ (chromospheric line). The spatial variations of the polarization will be used later to model the vertical structure of the turbulent magnetic field in the low chromosphere. From Fig. 9, we also derived profiles of $Q / I$ (see Fig. 11) for various limb distances $\left(0^{\prime \prime}, 5^{\prime \prime}, 10^{\prime \prime}, 20^{\prime \prime}\right.$ and $60^{\prime \prime}$ corresponding to $\mu=0.0,0.1,0.15,0.2$ and 0.35$)$. The polarization profile shows the same structure in the wings as previously observed by Stenflo \& Keller (1997): they attributed the hyperfine structure to odd isotopes, whereas the central peak is due to even isotopes. They measured polarization degrees of $1.2 \%$ at $\mu=$ 0.1 , which can be compared to ours $(0.6 \%)$ and other values of 

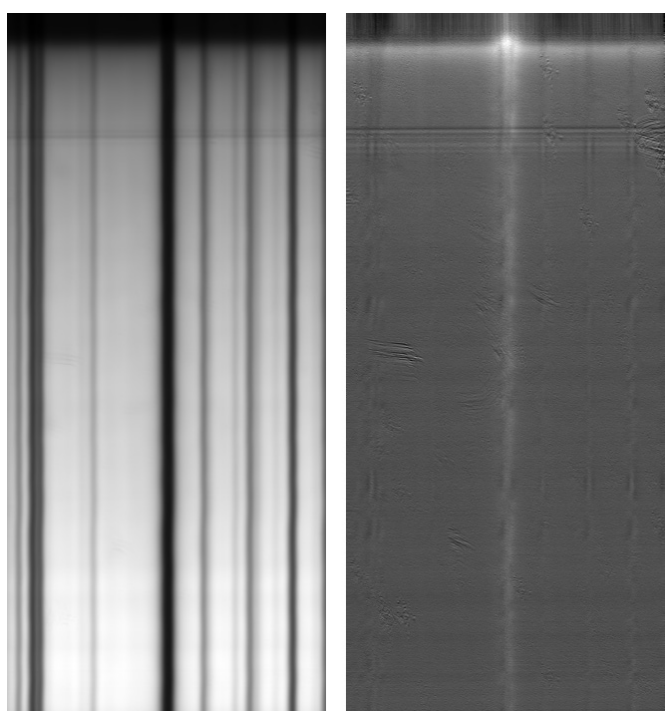

Fig. 9. I (left) and $Q / I$ (right) images of BaII $455.4 \mathrm{~nm}$. Abscissa: the wavelength (bandwidth of $0.35 \mathrm{~nm}$, pixel size of $1.1 \mathrm{pm}$ ); ordinates: the solar direction $x$ along the slit (field of view $140^{\prime \prime}$, pixel size $0.2^{\prime \prime}$ ).

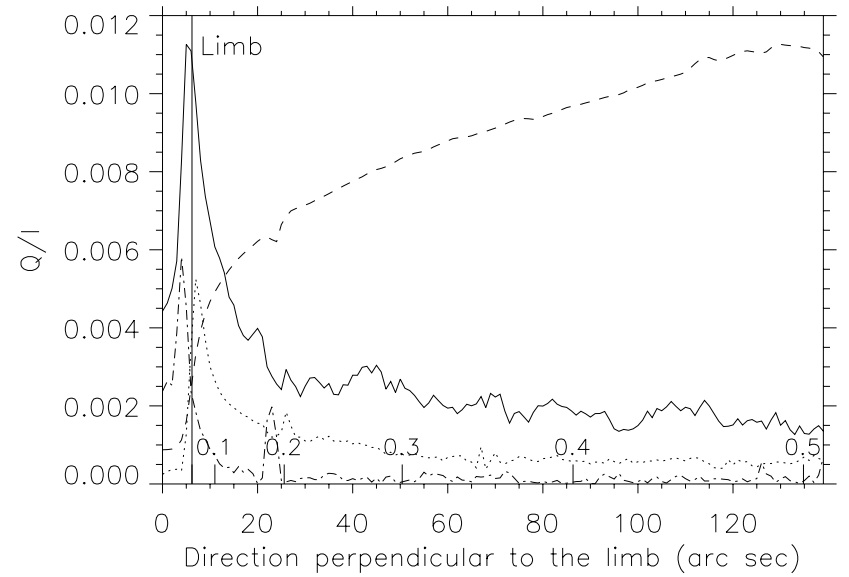

Fig. 10. $Q / I$ variations in the BaII $455.4 \mathrm{~nm}$ line centre (solid line) and in the continuum at $455.29 \mathrm{~nm}$ (dotted line) as a function of limb distance in arcsec (values of $\mu$ are indicated above $x$ axis). The continuum is integrated along 20 pixels in wavelength (bandwidth of $0.02 \mathrm{~nm}$ ). Default on the abscissa 25 is due to transversalium (dust or defaults along the slit of the spectrograph). The dashed line is the intensity profile (arbitrary units). The dashed dotted line represents the error bar of $Q / I$ which is of the order of $2 \times 10^{-4}$ except near the limb where it is limited to $2 \times 10^{-3}$.

Bommier \& Molodij (2000) and Gandorfer (2002), who found respectively $0.55 \%$ and $0.8 \%$ in the line core.

\section{Observations of the Cal $422.7 \mathrm{~nm}$ line}

Observations of the CaI $422.7 \mathrm{~nm}$ line were performed with the slit orthogonal to the limb on May 15, 2004. The core of this line is formed in the chromosphere. Observations were made at the West limb, and during $40 \mathrm{mn}, 2400$ pairs of images $I \pm Q$ were recorded, in order to reach a precision of $2 \times 10^{-4}$ in the line core for each pixel along the slit. Figure 12 shows the map $Q / I(\lambda, x)$ obtained after the processing of the 4800 spectra which were coaligned both in the spatial and the spectral directions. The limb is at the top of the figure. The line core and both wings show strong polarizations near the limb.

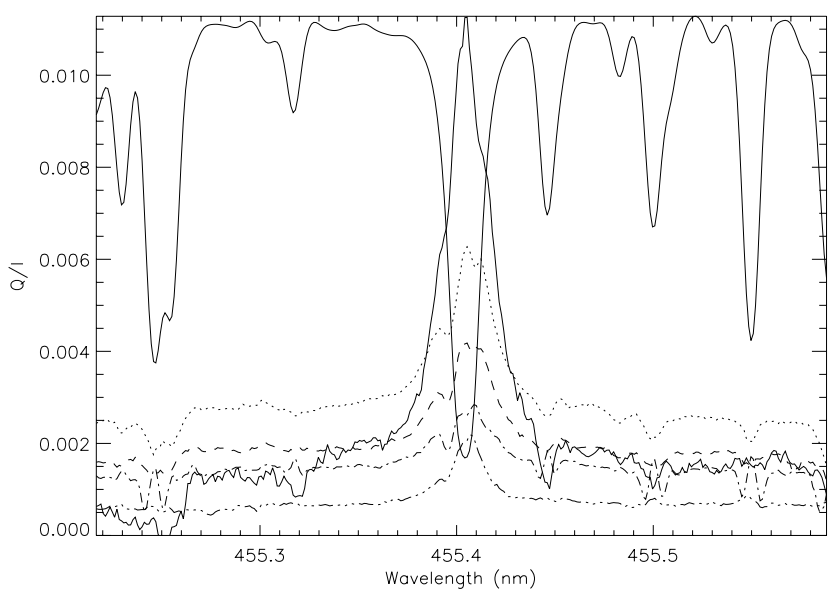

Fig. 11. $Q / I$ for the BaII $455.4 \mathrm{~nm}$ line at various distances of the limb $\left(0^{\prime \prime}, 5^{\prime \prime}, 10^{\prime \prime}, 20^{\prime \prime}\right.$ and $\left.60^{\prime \prime}\right)$ as a function of wavelength in $\mathrm{nm}$ (pixel size of $1.1 \mathrm{pm}$ ). The mean intensity curve (arbitrary units) is superimposed (thick line). The slit is orthogonal to the limb and data are averaged over $1^{\prime \prime}$.

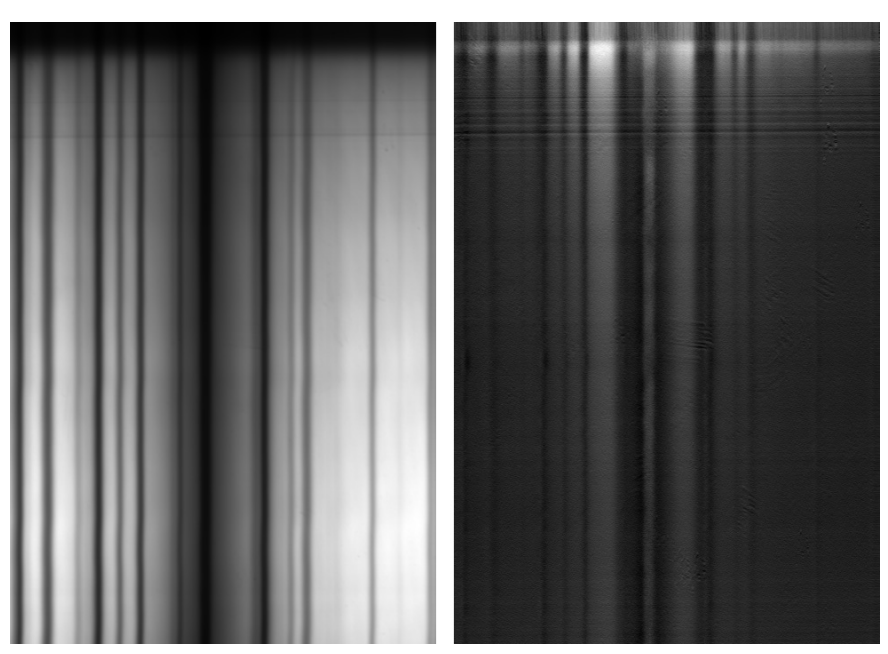

Fig. 12. I (left) and $Q / I$ (right) images of CaI $422.7 \mathrm{~nm}$. Abscissa: the wavelength (bandwidth of $0.35 \mathrm{~nm}$, pixel size of $1.0 \mathrm{pm}$ ); ordinates: the solar direction $x$ along the slit (field of view 140", pixel size $0.2^{\prime \prime}$ ).

From Fig. 12, we deduced the spatial variations of the polarization in the line core and in the continuum as a function of limb distance as shown in Fig. 13 where the pixel size was brought from $0.2^{\prime \prime}$ to $1.0^{\prime \prime}$ to improve the signal to noise ratio. The polarization rate near the limb was found to be $1.8 \%$ in the line core, $3.3 \%$ and $2.6 \%$ respectively in the blue and red wings, and $0.8 \%$ in the pseudo continuum at $422.92 \mathrm{~nm}$. The error bar due to image motion increases strongly from a few $0.01 \%$ to $0.2 \%$ at the limb. From Fig. 12, we also derived profiles of $Q / I(\lambda)$ (see Fig. 14) for various distances $\left(0^{\prime \prime}, 5^{\prime \prime}, 10^{\prime \prime}, 20^{\prime \prime}\right.$ and $80^{\prime \prime}$ corresponding to $\mu=0.0,0.1,0.15,0.2$, and 0.4$)$. At $\mu=0.1$, Gandorfer (2002) found near the South pole $2.2 \%$ in the red wing and a comparable value $(2.1 \%)$ in the core; in our data, the polarization in the core at $\mu=0.1(1.2 \%)$ is less than in the red wing $(1.8 \%)$. Center to limb variation of the polarization of CaI $422.7 \mathrm{~nm}$ has been studied by Bianda et al. (1998, 2003); they obtained similar polarization behaviors with highly fluctuating polarization degrees in the Doppler core (in the range $1.8 \%-3.6 \%$ at $\mu=0.1$ in quiet polar regions, down to $0.6 \%$ in an active region; in contrast the wings remained stable), and interpreted the spatial variation of this feature as the result of 


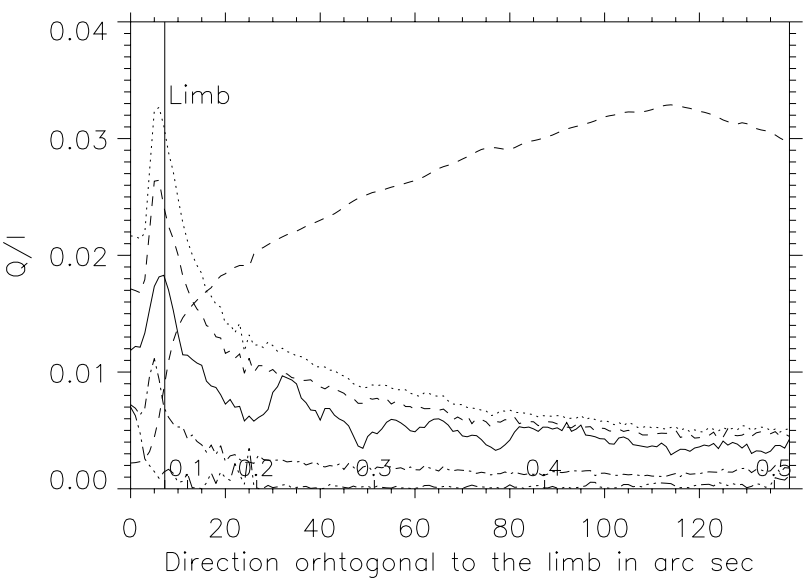

Fig. 13. $Q / I$ variations in the $\mathrm{CaI} 422.7 \mathrm{~nm}$ line centre (solid line), in the blue wing at $422.62 \mathrm{~nm}$ (dotted line), in the red wing at $422.72 \mathrm{~nm}$ (dashed line) and in the continuum at $422.92 \mathrm{~nm}$ (dashed dotted line) as a function of limb distance in arcsec (values of $\mu$ are indicated above $x$ axis). The intensity profile (arbitrary units) is also superimposed (dashed line). The decrease of intensities above $110^{\prime \prime}$ is an instrumental artefact. The dash dot dot line represents the error bar of $Q / I$ which is of the order of $2 \times 10^{-4}$ except near the limb where it is limited to $2 \times 10^{-3}$.

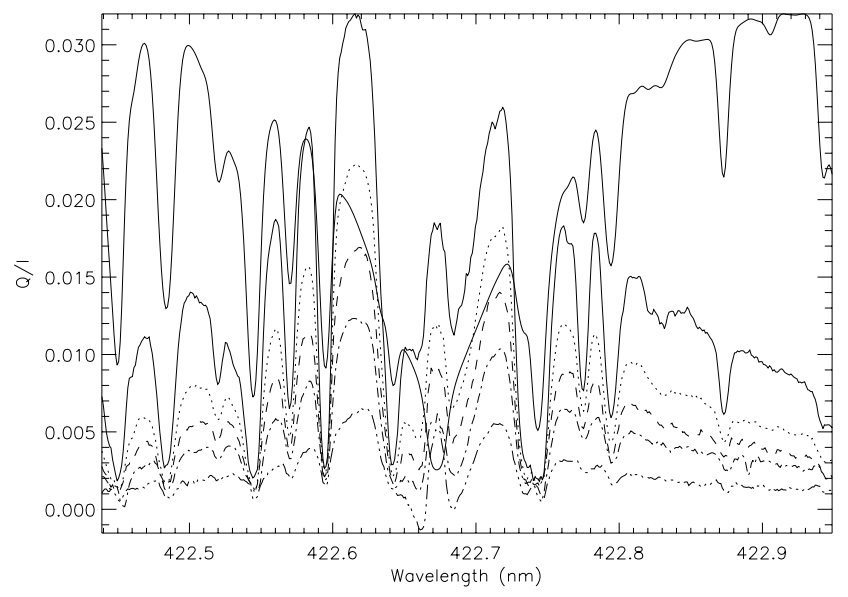

Fig. 14. $Q / I$ for the $\mathrm{CaI} 422.7 \mathrm{~nm}$ line at various distances of the limb $\left(0^{\prime \prime}, 5^{\prime \prime}, 10^{\prime \prime}, 20^{\prime \prime}\right.$ and $\left.80^{\prime \prime}\right)$ as a function of wavelength in nm (pixel size of $1.0 \mathrm{pm}$ ). The mean intensity curve is superimposed (thick line). The slit is orthogonal to the limb and data are averaged over $1^{\prime \prime}$.

Hanle depolarization due to the presence of magnetic fields of 5-15 G in the low to mid chromosphere. Bianda et al. (1999) measured Stokes $Q$ and $U$ and showed that the Hanle effect appears to be confined to the line core. But Bianda et al. (2003) discovered in some cases that depolarization and rotation of the plane of polarization may also occur in the wings. Holzreuter et al. (2005) and Fluri et al. (2006) suggested that the triplet peak structure of CaI $422.7 \mathrm{~nm}$ is mainly due to radiative transfer effects with partial frequency redistribution (PRD) effects.

\section{Polarimetry of the continuum around Srl, Ball and Cal lines in imagery mode}

The polarization of the continuum can be studied in spectroscopic mode, as a function of wavelength, but it can also be studied in imagery mode, through narrow $(0.3 \mathrm{~nm})$ interference filters, as done for instance by Wiehr \& Bianda (2003), or by Stenflo et al. (2002), with a very selective $(0.02 \mathrm{~nm})$
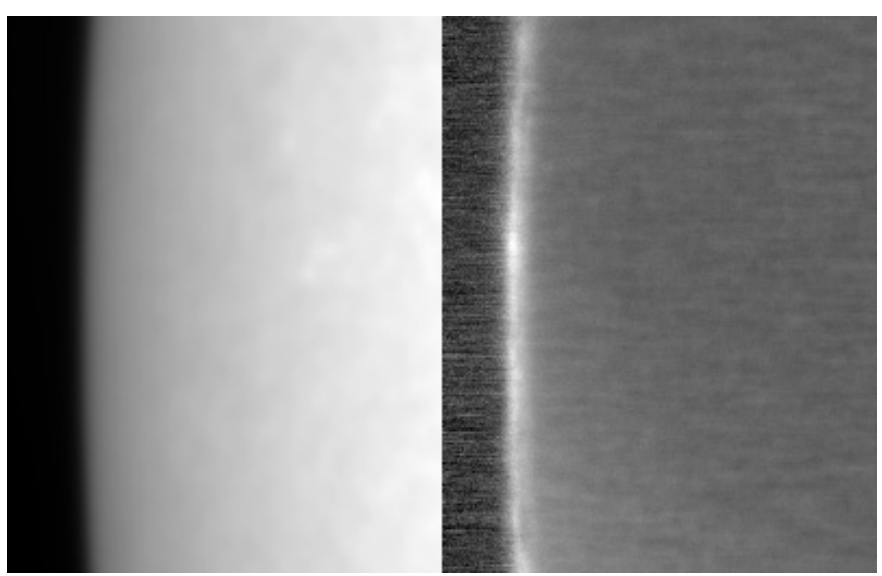

Fig. 15. Intensity $I(x, y)$ (left) and polarization $Q / I(x, y)$ images (right) of the limb through an interference filter of $10 \mathrm{~nm}$ bandwidth centered at $460 \mathrm{~nm}$, the field of view is about $100^{\prime \prime} \times 120^{\prime \prime}$.

Universal Birefringent Filter. This has the advantage, compared to spectroscopy, of allowing high spatial resolution polarimetry in a 2D field of view; the disadvantage is that the polarization is integrated in wavelength over the transmission function of the filter. Nevertheless, with narrow filters of $1 \mathrm{~nm}$ or less, precise measurements of the continuum polarization can be achieved, especially towards the red part of the spectrum. In our case, the filters are wide and comparable to those used by Leroy (1972) with $10 \mathrm{~nm}$ of full width at half maximum; we observed the blue part of the spectrum, so that many spectral lines are integrated and affect the results. However, these measurements can be related to the values obtained from spectroscopy and show the capability of our instrument to measure the continuum polarization. The instrumental setup of the polarimeter is identical to the one used in spectroscopy. The field of view is $100^{\prime \prime} \times 120^{\prime \prime}$ with a pixel size of $0.1^{\prime \prime}$, which was reduced to $0.2^{\prime \prime}$ by binning. The exposure time through $10 \mathrm{~nm}$ interference filters is of the order of $5 \mathrm{~ms}$ with a neutral density between 1 and 2, according to the spectral range. This means that exposure times would continue to be very short $(10 \mathrm{~ms})$ even with narrow filters (less than $1 \mathrm{~nm}$ ). For each observation, 2400 pairs of images $I \pm Q$ were recorded on October 26, 2005, near the West limb, in a quiet area, for about $15 \mathrm{mn}$, together with a flat field sequence of 800 pairs of images $I \pm Q$ at disk center. For each individual image, the flat field was successfully used to correct pixel response over the 2D field of view, and also to correct transmission differences between the two states of polarization. It also provided the zero polarization level. The limb position was tracked in the $x$ direction and the curvature of the limb was corrected. Image summation over the whole sequence and integration along the $y$ axis were performed in order to increase the signal to noise ratio. Figure 15 shows the final image got in the continuum at $460 \mathrm{~nm}$ around the SrI $460.7 \mathrm{~nm}$ line. This $Q / I$ image clearly shows the variation of the continuum polarization in the vicinity of the limb.

On October 26, 2005, we observed about 20 different spectral ranges from 390 to $670 \mathrm{~nm}$, but we present only here the results obtained in the continuum of spectral lines which are discussed in this paper. Figure 16 shows the polarization ratio $Q / I$ integrated in the $y$ direction (parallel to the limb) together with the intensity curve that gives the limb position (inflexion point). The error bar represents intensity fluctuations $(\Delta I / I)$ due to image motion corresponding to seeing-induced cross talk. Of course, values obtained closer than $1^{\prime \prime}$ from the limb have to be regarded with caution. The polarization degree $Q / I$ reaches 


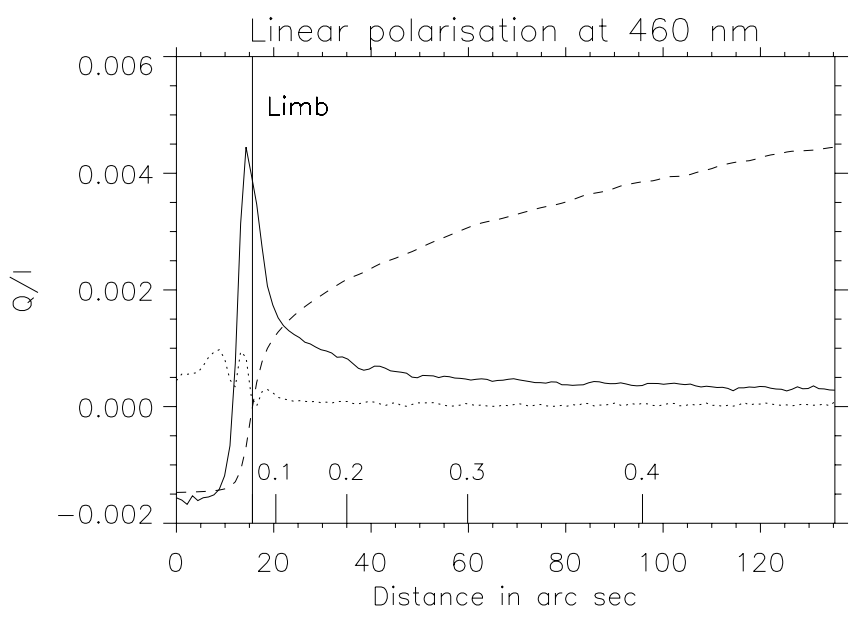

Fig. 16. Polarization rate $Q / I$ of the continuum through an interference filter of $10 \mathrm{~nm}$ bandwidth centered at $460 \mathrm{~nm}$, as a function of limb distance in arcsec (values of $\mu$ are indicated above $x$ axis). Solid line: $Q / I$. Dashed line: the intensity profile (arbitrary units), which defines the limb position (inflexion point). Dotted line: the error bar of $Q / I$, which increases at the limb $(0.1 \%)$.

$0.35 \%$ at the limb and $0.16 \%$ at $\mu=0.1$. The measure of the continuum polarization is very difficult at the limb, for at least two reasons: the polarization is much less than in the core of polarized spectral lines, and the intensity drops at the limb and is affected by atmospheric turbulence. Thus, the error bar increases towards $0.1 \%$ at the limb (located at $x=16^{\prime \prime}$ ), so that below the inflexion point, the result is meaningless. However, it remains in agreement with the values obtained in the neighborhood of SrI $460.7 \mathrm{~nm}$ in spectroscopic mode $(Q / I=0.5 \%$ at the limb and $Q / I=0.2 \%$ at $\mu=0.1$, over a narrow bandwidth of $0.02 \mathrm{~nm}$, see Fig. 6).

Figure 17 shows results around $455 \mathrm{~nm}$. The polarization degree $Q / I$ reaches $0.35 \%$ at the limb and $0.18 \%$ at $\mu=0.1$, which is in fairly good agreement with the determination using the previous filter at $460 \mathrm{~nm}$. At the limb, located at $x=18^{\prime \prime}$, the error barr is $0.1 \%$. As this spectral range contains the BaII $455.4 \mathrm{~nm}$ line, we can compare to the results of Fig. 10 which give $Q / I=$ $0.5 \%$ at the limb and $Q / I=0.2 \%$ at $\mu=0.1$ over a bandwidth of $0.02 \mathrm{~nm}$.

We found through the wide $10 \mathrm{~nm}$ bandwidth filters polarization degrees smaller than those obtained by spectroscopic means $(0.45 \%$ if the offset suspected previously is subtracted), or through narrow filters, as reported by Wiehr \& Bianda (2003): they got $0.45 \%$ close to the limb $\left(0.3^{\prime \prime}\right.$ or $\left.\mu=0.02\right)$ with narrow $(0.3 \mathrm{~nm})$ filters at $450 \mathrm{~nm}$, in agreement with the theoretical polarization continuum model of Fluri \& Stenflo (1999). We suggest that our underestimation could be explained by the presence, in the blue part of the spectrum, of numerous spectral lines that depolarize the continuum, as discussed by Fluri \& Stenflo (2003). The polarization of the continuum was also modelled by Stenflo (2005) using data from the second solar spectrum atlas (Ganddorfer 2002). At $\mu=0.1$, the model gives at $450 \mathrm{~nm}$ values around $0.1 \%$, somewhat smaller than the ones obtained by us and by Wiehr \& Bianda (2003).

Figure 18 shows results around $420 \mathrm{~nm}$. The polarization degree $Q / I$ reaches $0.53 \%$ near the limb and $0.40 \%$ at $\mu=0.1$. At the limb, located at $x=13^{\prime \prime}$, the error bar is again $0.1 \%$. As this spectral interval contains the $\mathrm{CaI} 422.7 \mathrm{~nm}$ line, we can compare the imagery data to the spectroscopic results of Fig. 13 which give $Q / I=1 \%$ at the limb and $Q / I=0.4 \%$ at $\mu=0.1$

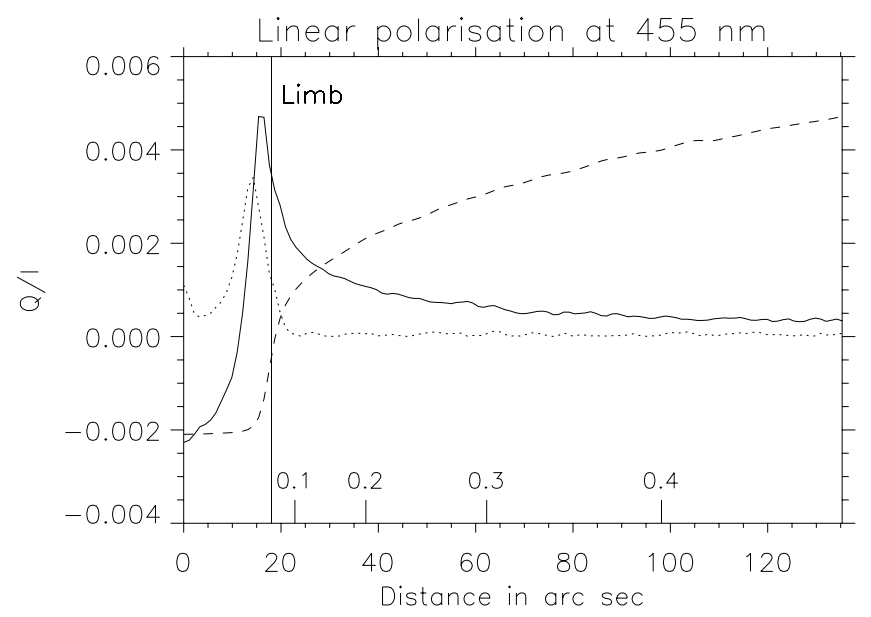

Fig. 17. Polarization rate $Q / I$ of the continuum through an interference filter of $10 \mathrm{~nm}$ bandwidth centered at $455 \mathrm{~nm}$, as a function of limb distance in arcsec (values of $\mu$ are indicated above $x$ axis). Solid line: $Q / I$. Dashed line: $I$ (arbitrary units). Dotted line: the error bar of $Q / I$, which increases at the limb $(0.1 \%)$.

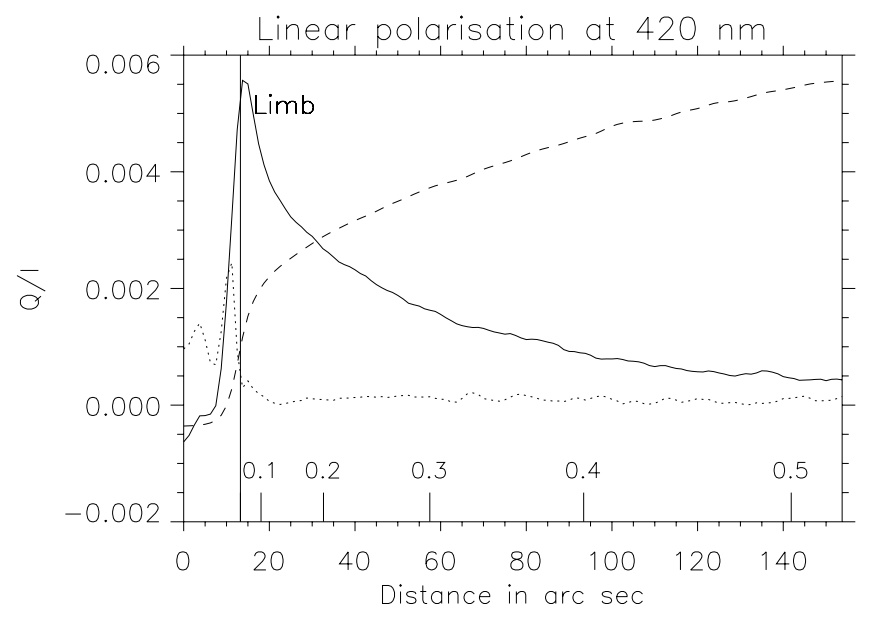

Fig. 18. Polarization rate $Q / I$ of the continuum through an interference filter of $10 \mathrm{~nm}$ bandwidth centered at $420 \mathrm{~nm}$, as a function of limb distance in arcsec (values of $\mu$ are indicated above $x$ axis). Solid line: $Q / I$. Dashed line: $I$ (arbitrary units). Dotted line: the error bar of $Q / I$, which increases at the limb $(0.1 \%)$.

for the pseudo continuum at $422.92 \mathrm{~nm}$. The atlas of Gandorfer gives in comparison $0.55 \%$ at $\mu=0.1$ for the same wavelength. This is higher than values predicted by Fluri \& Stenflo (1999) or by Stenflo (2005) for the continuum with respectively $0.25 \%$ and $0.20 \%$. This discrepancy is easily explainable: first, the polarization observed through the filter is affected by the presence of depolarizing lines and the strong polarized region around the $\mathrm{CaI}$ line; secondly, the spectroscopic measure at $422.92 \mathrm{~nm}$ is overestimated because, at this wavelength, we are still in the very broad wings of the $\mathrm{CaI} 422.7 \mathrm{~nm}$ line.

\section{Observations of the Nal D1 $589.6 \mathrm{~nm}$ line}

Observations of the NaI D1 $589.6 \mathrm{~nm}$ spectral line have been reported by Stenflo \& Keller (1997) and Stenflo et al. (2000a,b) as anomalous polarization, with a polarization peak in the Doppler core. They found with ZIMPOL a polarization rate around $0.1 \%$ at $5^{\prime \prime}$ from the limb. NaI D1 is theoretically unpolarizable, unlike NaI D2 $589.0 \mathrm{~nm}$; quantum interference 


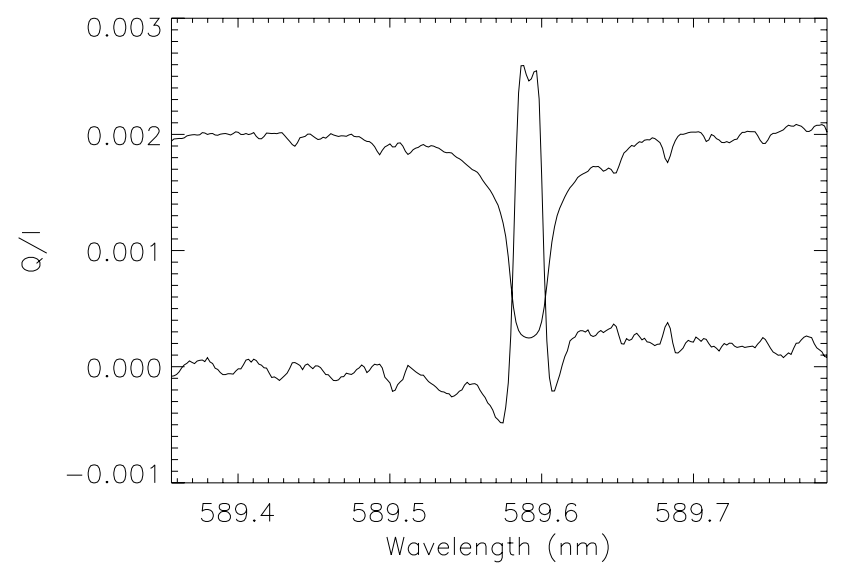

Fig. 19. $Q / I$ profile of the NaI D1 $589.6 \mathrm{~nm}$ line as a function of wavelength (in $\mathrm{nm}$ ), together with the intensity profile (arbitrary units). Polarization fringes are partly corrected.

between both transitions was investigated successfully to explain the overall polarization pattern. Although it has been demonstrated that fine and hyperfine structure (splitting due to nuclear spin) in combination with optical pumping must play a role in the scattering polarization in the sodium lines (Landi 1998; Casini \& Manso Sainz 2005), the nature of the Na D1 line polarization still remains an enigma. Other anomalous polarizations corresponding to D1 transitions of the same class $(J=$ $\frac{1}{2} \rightarrow \frac{1}{2} \rightarrow \frac{1}{2}$ ) were also discovered by Stenflo et al. (2000a), as BaII $493.4 \mathrm{~nm}$, SiII $637.1 \mathrm{~nm}$, AlI $669.8 \mathrm{~nm}$, VI $6111.1 \mathrm{~nm}$ or LiI $670.8 \mathrm{~nm}$.

We performed new observations of the weak polarization of NaI D1 $589.6 \mathrm{~nm}$ on July 9, 2004 at $10^{\prime \prime}(\mu=0.15)$ of the South pole, with the slit $\left(0.6^{\prime \prime} \times 140^{\prime \prime}\right)$ parallel to the limb direction, and an exposure time of $20 \mathrm{~ms}$. Several series of 1600 pairs of images $I \pm Q$ were obtained and we selected the best one. The precision obtained in the line core, after integration along the slit, was about $2.5 \times 10^{-5}$. The result is displayed in Fig. 19. A polarization peak in the line core appears clearly (maximum $0.25 \%$ ) and corroborates previous results. Stenflo et al. (2000b) reported observations showing that the polarization of the NaI D1 core may vary in the range $0.1 \%$ to $0.25 \%$ in time and also according to the location on the sun. Martinez Pillet et al. (2001) noticed also spatial variations. In agreement with Stenflo et al. (2000a,b), the polarization peak of Fig. 19 does not have the characteristic antisymmetric shape of scattering polarization, which suggests, according to Trujillo Bueno et al. (2002) the presence of weak magnetic fields. On the contrary, Bommier \& Molodij (2002) found at THEMIS a pure antisymmetric polarization shape with respect to the line center with $Q / I$ smaller than $0.1 \%$.

Figure 19 shows that observations are slightly affected by polarization fringes. They are very hard to remove because the pattern appearing on flat field images at disk center is not the same, so that fringes do not match. It was only possible to attenuate them. We averaged the ratio $Q / I(\lambda, x)$ along the solar direction for observations and flat field, in order to detect the shift of the fringe network by cross correlation. Then the fringe pattern of the flat field was subtracted from the limb data. We succeeded in attenuating fringes by a factor of two.

\section{Conclusion}

We have presented the new polarimetric instrumentation operating since 2004 at the Lunette Jean Rösch, Pic du Midi, in spectroscopic or imagery mode, allowing one to sequentially measure the whole set of Stokes parameters using Nematic Liquid Crystal cells. The present version of this polarimeter has still some limitations which will be removed in the future. In particular, polarization signals $I \pm P(P=Q, U, V)$ are analyzed sequentially with a linear polarizer. A new analyser is under development, consisting of a birefringent linear polarizing beam splitter which will allow one to simultaneously observe both signals, so that $I \pm P$ will be focused at the same time on the $\mathrm{CCD}$ detector. As a consequence, the field of view will be reduced by a factor of two, from $140^{\prime \prime}$ to $70^{\prime \prime}$ along the sun. This improvement is of special importance for the polarimetry of specific solar structures observed on the disk (active regions, flux tubes) or at the limb (spicules, prominences) which require perfect coalignment of both signals, in order to eliminate seeing induced polarimetric cross talk. But observations of the second solar spectrum do not suffer from this default, because of statistical effects due to the large number (thousands) of spectra required to achieve a high enough signal to noise ratio. Beam exchange will be available with the new device especially for the measurement of weak polarization signals.

Observations of the second solar spectrum have been undertaken with the new polarimeter. We present observations of the linear polarization $Q / I$ with a spatial resolution of $\operatorname{SrI} 460.7 \mathrm{~nm}$, BaII $455.4 \mathrm{~nm}$ and $\mathrm{CaI} 422.7 \mathrm{~nm}$ lines and show spatial variations of the polarization through the limb. The polarization rate at the limb reaches $1.8 \%$ and $1.2 \%$ in the core of SrI and BaII, and more than $2 \%$ in the core (up to $3 \%$ in the wings) of CaI. Such results concerning the variation of the linear polarization as a function of limb distance are of interest in probing the variation of weak turbulent magnetic fields as a function of depth in the solar atmosphere. Spatial variations of SrI $460.7 \mathrm{~nm}$ were investigated at $40^{\prime \prime}$ from the limb, and preliminary results show that dark regions (intergranules) could be less polarized than bright regions (granules), suggesting stronger magnetic fields in intergranular lanes. Continuum polarization was measured in the vicinity of lines, and also in imagery mode, through wide $(10 \mathrm{~nm})$ interference filters. Finally, results of observations of weak polarizations are illustrated by the NaI D1 line with the confirmation of a symmetric polarization peak. These results have been obtained in general in poor seeing conditions; some aspects of the spatial variations of the second solar spectrum, in particular the distribution of the polarization in the photospheric and chromospheric network, require new observations at higher spatial resolution, together with the determination of the Stokes $U$ parameter. This will be the topic of further work.

Acknowledgements. This paper is the result of two years of tests and observing campaigns. We thank the anonymous referee who provided helpful and detailed comments and suggestions. We are indebted to Ch. Coutard, engineer in optics, for his help in the optical setup of the experiment and his support in observations. The spectrograph and the MSDP device were designed by Z. Mouradian and by P. Mein under the auspices of Observatoire de Paris and CNRS. This work was supported by Observatoire de Paris, Observatoire Midi Pyrénées, CNRS (UMR 8109 and UMR 5572) and the Programme National Soleil Terre (PNST). Special thanks are due to the Pic du Midi Observatory staff for their technical assistance during our numerous stays.

\section{References}

Bianda, M., Solanki, S. K., \& Stenflo, J. O. 1998, A\&A, 331, 760

Bianda, M., Stenflo, J. O., \& Solanki, S. K. 1999, A\&A, 350, 1060

Bianda, M., Stenflo, J. O., Gandorfer, A., \& Gisler, D. 2003, ASP Conf. Ser., 286, 61

Bommier, V., \& Molodij, G. 2002, A\&A, 381, 241

Bommier, V., Derouich, M., Landi Degl'Innocenti, E., Molodij, G., \& Sahal-Bréchot, S. 2005, A\&A, 432, 295 
Casini, R., \& Manso Sainz, R. 2005, ApJ, 624, 1025

Chen, W., Chiang, C., \& Nee, J. B. 2002, Appl. Opt., 41, 6470

Derouich, M., Bommier, V., Malherbe, J. M., \& Sahal-Bréchot, S. 2006, A\&A, 457, 1047

Faurobert, M., Arnaud, J., Vigneau, J., \& Frish, H. 2001, A\&A, 378, 627

Fluri, D., \& Stenflo, J. O. 1999, A\&A, 341, 902

Fluri, D., \& Stenflo, J. O. 2003, A\&A, 398, 763

Fluri, D., Holzreuter, R., \& Stenflo, J. O. 2006, ASP Conf. Ser., in press

Gandorfer, A. 2000, The second solar spectrum, Vol 1, $4625 \AA$ to $6995 \AA$ A Zurich VDF

Gandorfer, A. 2002, The second solar spectrum, Vol 2, 3910 Å to $4630 \AA$ A Zurich VDF

Holzreuter, R., Fluri, D., \& Stenflo, J. O. 2005, A\&A, 434, 713

Leroy, J.-L. 1972, A\&A, 19, 287

Landi Degl'Innocenti, E. 1998, Nature, 392, 256

Malherbe, J.-M., Roudier, Th., Mein, P., Moity, J., \& Muller, R. 2004, A\&A, 427,745

Martinez Pillet, V., Trujillo Bueno, J., \& Collados, M. 2001, ASP Conf. Ser., 236, 133

Mein, P. 2002, A\&A, 381, 271

Mouradian, Z., Chauveau, F., Colson, F., et al. 1980, Japan France Seminar on Solar Physics, 271

Noel, V., Vinker, D., McGill, M., \& Lawson, P. 2004, J. Geophys. Res., 109, D24213
Noel, V., Chepfer, H., Ledanois, G., Delaval, A., \& Flamant, P. 2002, Appl. Opt., 41,4245

Roudier, T., Malherbe, J. M., Moity, J., Mein, P., \& Coutard, C. 2006, A\&A, 455, 1091

Sanchez Almeida, J. 2005, A\&A, 438, 727

Stenflo, J. O. 2003a, Spectro polarimetry, in The Dynamic Sun, ed. Dwivedi (Cambridge Univ. Press), 128

Stenflo, J. O. 2003b, in Solar Polarization, ed. Trujillo Bueno, \& Sanchez Almeida, Proc. 3rd SPW, ASP Conf. Ser., 307, 385

Stenflo, J. O. 2004, Rev. Mod. Astron., 17, 269

Stenflo, J. O. 2005, A\&A, 429, 713

Stenflo, J. O. 2006, Proc. 4rd SPW, ASP Conf. Ser., in press

Stenflo, J. O., \& Keller, C. U. 1997, A\&A, 321, 927

Stenflo, J. O., Twerenbold, D., \& Harvey, J. W. 1983a, A\&AS, 52, 161

Stenflo, J. O., Twerenbold, D., Harvey, J. W., \& Brault, J. W. 1983b, A\&AS, 54, 505

Stenflo, J. O., Bianda, M., Keller, C. U. \& Solanki, S. K. 1997, A\&A, 322, 985

Stenflo, J. O., Gandorfer, A., \& Keller, C. U. 2000a, A\&A, 355, 781

Stenflo, J. O., Keller, C. U., \& Gandorfer, A. 2000b, A\&A, 355, 789

Stenflo, J. O., Gandorfer, A., Holzreuter, R., et al. 2002, A\&A, 389, 314

Trujillo Bueno, J., Shchukina, N., \& Ascensio Ramos, A. 2004, Nature, 430, 326

Trujillo Bueno, J., Casini, R., Landolfi, M., \& Landi, E. 2002, ApJ, 566, L53

Wiehr, E., \& Bianda, M. 2003, A\&A, 398, 739 


\section{Online Material}



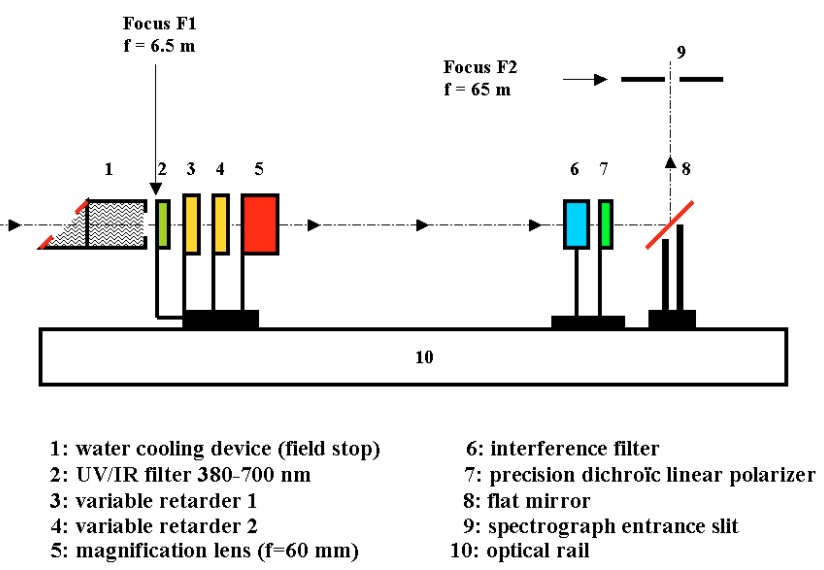

Fig. A.1. The optical setup between the primary focus F1 and the secondary focus F2 in spectroscopic mode (the $50 \mathrm{~cm}$ refractor is located on the optical axis at left).

\section{Appendix A: The experiment setup in spectroscopic mode}

The Pic du Midi LJR is a $50 \mathrm{~cm}$ aperture refractor (focal length of $6.45 \mathrm{~m}$ for $\lambda=550 \mathrm{~nm}$ at the primary focus $\mathrm{F} 1$ ) on an equatorial mount. The beam has an axial symmetry along the optical axis. Polarization analysis is achieved before transmission to the spectrograph by a flat mirror at $45^{\circ}$ (Fig. A.1), therefore instrumental polarization is minimized. The spatial resolving power (including the refractor and the spectrograph) is $0.3^{\prime \prime}$ in the yellow part of the spectrum. The primary image is magnified 10 times at the secondary focus F2 where the slit of the spectrograph is located, according to the magnification lens, providing an equivalent focal length of $65 \mathrm{~m}$ so that the spectrograph operates at $f / 130$. We used a new prototype of a liquid crystal polarimeter between focus F1 and F2. It was used for the first time in April 2004 in a simplified version and upgraded to the full Stokes version in September 2004.

The polarimeter receives white light and has the following elements (Fig. A.1):

- Two variable retarders (retardance continuously adjustable between 0 and $700 \mathrm{~nm}$ ) made by Meadowlark (USA) which combine a single Nematic Liquid Crystal (NLC) cell with a static birefringent element (Fig. A.2). A $2 \mathrm{KHz}$ square wave signal with zero mean value is applied to the crystal; the amplitude of the signal $(0$ to $10 \mathrm{~V})$ determines the retardance. The maximum birefringence (so the maximum retardance) is obtained for an amplitude of $0 \mathrm{~V}$ and decreases continuously with increasing voltage (no retardance at about $6 \mathrm{~V}$ ). Consequently, a variable retarder can be exactly quarter or half wave for any wavelength according to the voltage applied to the electrodes. The calibration was performed in the laboratory using a white light source, various interference filters of $10 \mathrm{~nm}$ bandwidth, with a 10 bits CMOS camera used as a detector and mounted on an optical rail (Fig. A.3). It takes about $25 \mathrm{~ms}$ for the retarder to switch between zero to half wave using the fast transient nematic effect: in order to speed up the response of the crystal, a high to low voltage transition is preceded by a short $(5 \mathrm{~ms}) 0 \mathrm{~V}$ pulse, while a low to high voltage transition is preceded by a $10 \mathrm{~V}$ pulse. The NLC is protected by a pass band filter in order to avoid damage of the crystal by the UV radiation (below $380 \mathrm{~nm}$ ), and heating by IR radiation (above $700 \mathrm{~nm}$ ). The temperature of the two liquid crystals is controlled permanently

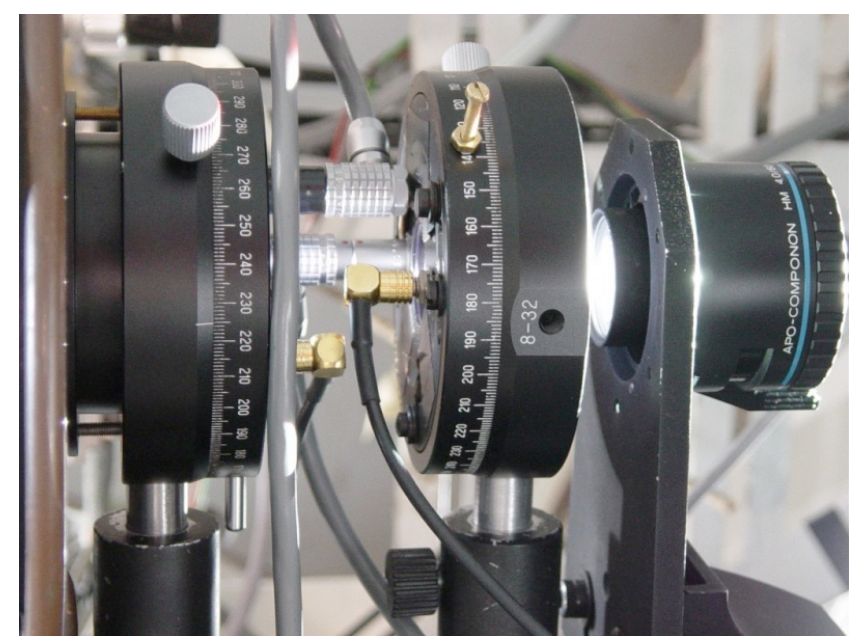

Fig. A.2. The two variable retarders (at left) and the magnifying lens (at right) in the beam near focus F1 with wires for temperature control and modulation.

(active heating by resistive elements and passive cooling) and is ordinarily set to $20^{\circ} \mathrm{C}$.

- A precision dichroic linear polarizer from Meadowlark (USA) is located at the exit of the system, on the optical axis, before the reflecting mirror to the spectrograph. The acceptance axis is orthogonal to the long direction of the entrance slit in order to be orthogonal to the rules of the Echelle grating of the spectrograph (maximum luminosity). The precision of the polarizer is of the order of $10^{-5}$ (this value represents light transmission through two identical crossed polarizers). The fast axis of the first retarder is parallel to the acceptance axis of the polarizer, while the fast axis of the second retarder makes an angle of $45^{\circ}$.

\section{A.1. Version of the polarimeter with two retarders}

The signal $S$ provided by the polarimeter with two NLC is given by:

$S=\left(\frac{1}{2}\right)(I+Q \cos \delta 2+\sin \delta 2(U \sin \delta 1-V \cos \delta 1))$

where $I, Q, U$ and $V$ are the Stokes parameters defined by the relations:

$I=A \bar{A}+B \bar{B}$

$Q=A \bar{A}-B \bar{B}$

$U=\bar{A} B+A \bar{B}$

$V=\mathrm{i}(\bar{A} B-A \bar{B})$

$A$ and $B$ are the complex amplitudes of the electric field (the conjugate quantity is denoted by the bar) $\vec{E}=\left(A \mathrm{e}^{\mathrm{i} \omega t}, B \mathrm{e}^{\mathrm{i} \omega t}, 0\right), \omega=$ $2 \pi / v, v$ frequency of the light, in a reference frame where the $x$ axis is the geographic East West direction, the $y$ axis is the geographic North South direction of the equatorial mount of the refractor, and the $z$ direction is the optic axis. $\delta 1, \delta 2$ are the retardances of the two NLC. When the first retarder is set to 0 wave $(\delta 1=0)$, and when the second retarder modulates between 


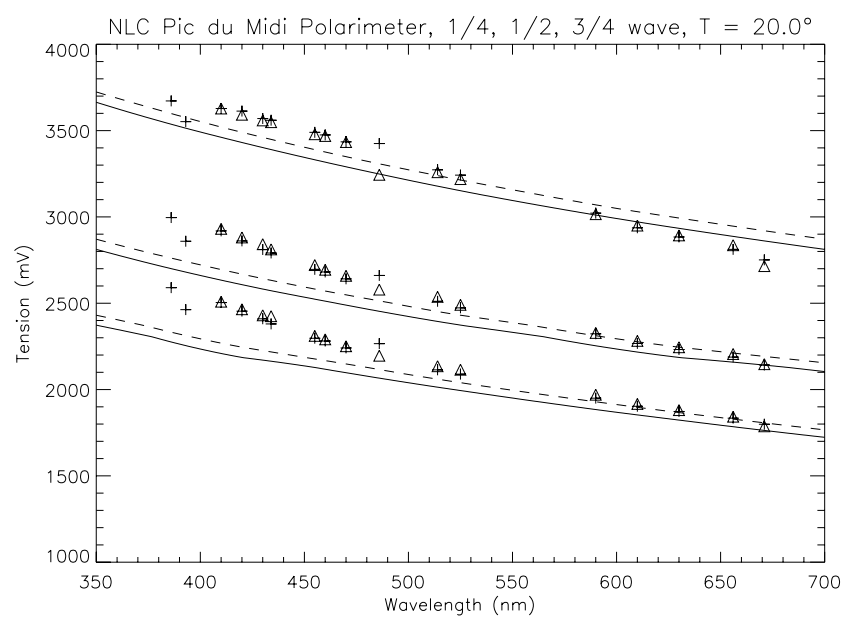

Fig. A.3. The voltage applied to the two NLC as a function of wavelength for quarter (top), half (middle), or three quarter (bottom) wave retardance. Crosses and triangles indicate our calibration points obtained with various interference filters ( $10 \mathrm{~nm}$ bandwidth) for the two retarders at $386,393,410,420,430,434,455,460,470,486,514,523,590,610$, 630,656 and $670 \mathrm{~nm}$. For comparison, the two curves were extrapolated from data provided by the manufacturer.

quarter $(\delta 2=\pi / 2)$ and three quarter wave $(\delta 2=3 \pi / 2)$, we sequentially get $I \pm V$; when it modulates between $0(\delta 2=0)$ and half wave $(\delta 2=\pi)$, we obtain $I \pm Q$. In order to measure $I \pm U$, the first retarder must be set to quarter wave $(\delta 1=\pi / 2)$, while the second retarder has to modulate between quarter $(\delta 2=\pi / 2)$ and three quarter waves $(\delta 2=3 \pi / 2)$. In our reference frame, the angle $\alpha$ of the polarization direction with $x$ axis is given by $\alpha=\left(\frac{1}{2}\right) \arctan \left(\frac{U}{Q}\right)$ so that $Q$ characterizes schematically the linear polarization parallel or orthogonal to $x$ axis $(A=0$ or $B=0$ gives $U=0$ ), while $U$ is related to the linear polarization at $\pm 45^{\circ}$ of $x$ axis $(A= \pm B$ implies $Q=0)$. $V$ as usual characterizes the circular polarization.

\section{A.2. Version of the polarimeter with one retarder}

We use for observations which do not require the full Stokes configuration a simplified version of the polarimeter, running only with one retarder (no possible $U$ determination). In such a configuration ( $\delta 1$ is permanently set to zero), the polarimeter allows in practice modulation from zero to three quarter waves, providing analysis either of the circular or linear polarization of light. Hence, Stokes parameters $I \pm V$ are obtained sequentially from the output signal:

$S=\left(\frac{1}{2}\right)(I+Q \cos \delta-V \sin \delta)$

where $\delta=(\pi / 2,3 \pi / 2)$ is the retardance of the NLC. This property was used by Roudier et al. (2006) for high resolution Zeeman magnetometry on the disk. In the present paper, we are interested only in $I \pm Q$ which were derived from this formula with $\delta=(0, \pi)$.

Since measurements of $I \pm P$ (where $P$ is any Stokes parameter) are not simultaneous, our polarimeter can operate with a good efficiency in two particular domains:

- observations of the line of sight magnetic fields on the disk, with the 2D imaging spectroscopy device provided by the Multichannel Subtractive Double Pass (MSDP), as described by Malherbe et al. (2004) and Mein (2002); with a 2D field of view, we use cross correlation and destretching methods to superimpose properly solar structures observed sequentially in two states of circular polarization $(I \pm V)$;

- observations of the second solar spectrum near the limb; since the polarization degrees are small (less than $1 \%$ ), the signal has to be derived from the statistical analysis of hundreds or thousands of spectra obtained sequentially, in order to improve the signal to noise ratio. Consequently, non simultaneous measurements of $I \pm Q$ have a moderate impact on the final polarization rates. Since the spectrograph has a good transmission in the blue part of the spectrum, and the CCD detector has the maximum quantum efficiency (65\%) around 400-500 nm, our observations are mainly focused in this range of the second solar spectrum. We started observations with two scientific goals:

1. measurements of weak polarizations $(0.01 \%)$ at moderate spatial resolution: when the seeing conditions are not excellent, we use a $0.6^{\prime \prime} \times 140^{\prime \prime}$ slit and accumulate a large number of spectra to achieve the best polarimetric sensitivity as possible; data can be averaged partially or totally along the slit to reduce the noise.

2. measurements of stronger polarizations $(0.1 \%$ to $1 \%)$ at higher spatial resolution, as in the SrI $460.7 \mathrm{~nm}$ line: when the seeing conditions are fairly good, we use a thin slit of $0.3^{\prime \prime} \times 140^{\prime \prime}$ in order to select structures along the slit, in terms of bright and dark regions. Such a diagnostic is theoretically able to bring new information about turbulent unresolved magnetic fields in the structures of the quiet sun.

\section{A.3. The spectrograph}

We used the $8 \mathrm{~m}$ Littrow Echelle spectrograph (Fig. A.4) built by Paris Observatory at the LJR twenty years ago and modified later for 2D spectro imagery (MSDP). The dispersive element is a grating ( 316 rules $/ \mathrm{mm}$, blaze angle $63^{\circ} 26^{\prime}$ ) that provides a typical dispersion of $50 \mathrm{~mm}$ per $\mathrm{nm}$ at the focus of the spectrograph. The interference order is isolated by filters of typically $10 \mathrm{~nm}$ bandwidth. The spectrum obtained at the focus of the spectrograph is reduced to form on a CCD camera from LaVision (Germany) with temperature control (Peltier cooling at $-10{ }^{\circ} \mathrm{C}$ ). This is a shutterless interline scan camera using a detector with microlenses manufactured by Sony $(1376 \times 1040,6.45 \mu$ square pixels). The spatial pixel size on the CCD is $0.2^{\prime \prime}$ along the slit direction and the spectral pixel size around $1.1 \mathrm{pm}$ (in the blue part of the spectrum) in our setup. Each pixel can accumulate up to 20000 electrons corresponding to a dynamic of 12 bits (readout noise of 4-5 electrons, gain of 4 electrons per analog digital unit). In general, we work in the continuum at 0.8 times the saturation level giving approximately a signal to noise ratio of 120 , corresponding to a photometric accuracy of $0.8 \times 10^{-2}$. In the core of the lines (smaller number of photons), this precision can drop to about $1.6 \times 10^{-2}$. The exposure time is typically $50 \mathrm{~ms}$ during our runs around $450 \mathrm{~nm}$ at $\mu=0.15$ (limb distance of $10^{\prime \prime}$ ) with the $0.6^{\prime \prime}$ slit.

\section{A.4. Signal to noise ratio}

Our polarimetric observations consist of shooting sequentially as fast as possible pairs of images $I \pm Q$ (typically 700 pixels in the solar direction $x 300$ pixels in the spectral direction). The polarimeter is able to run at $40 \mathrm{~Hz}$, but in practice we are limited by exposure times and by the readout speed of our CCD camera 


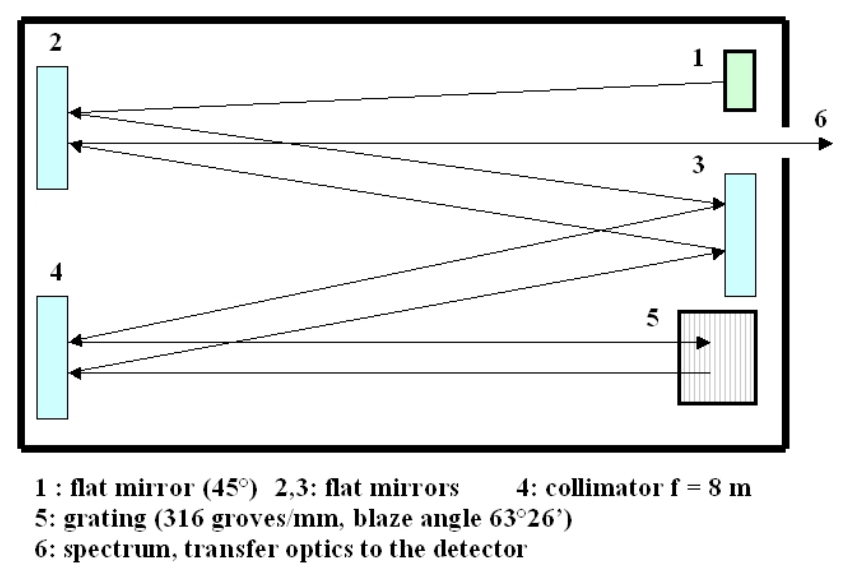

Fig. A.4. The $8 \mathrm{~m}$ Littrow Echelle spectrograph.

and data acquisition system, so that the maximum speed is at best $10 \mathrm{~Hz}$ around $450 \mathrm{~nm}$, including polarimetric modulation.

Since a pair of images $I \pm Q$ has a photometric precision of about $10^{-2}$, one hour of observation at the limb at $450 \mathrm{~nm}$ allows us to reach roughly $10^{-4}$ with the average throughput of 5 images/s. It is even possible to attain weaker polarization signals by integrating partially or totally in the solar direction along the slit (700 pixels). In the case of total integration, a single pair of images $I \pm Q$ will deliver a precision better than $10^{-3}$, and after one hour of observations, a ratio better than $10^{-5}$ can be achieved.

\section{A.5. Flat Field and data processing}

Flat field observations are made around disk center in the quiet sun where linear polarization signals should vanish. But good flat fielding is a difficult challenge at the LJR because the spectrograph is attached to the refractor (in equatorial mount) and moves with time, producing very small but permanent mechanical shifts. For that reason, the spectral line has to be precisely tracked by the software at sub pixel precision in the direction of dispersion. For observations on the disk, the transversalium is followed in the direction of the slit, while for observations with the slit perpendicular to the limb, the position of the limb itself is detected by the software with about $1^{\prime \prime}$ accuracy, depending on seeing. The flat field is consequently never done exactly in the same conditions than the observing run. In particular, polarization fringes cannot be properly corrected by the flat field procedure. They are not important for polarizations in the range $10^{-3}$ to $10^{-2}$ but have to be considered carefully for polarizations of $10^{-4}$ or smaller. We have two kinds of fringes: filter fringes (mainly due to the interference filter) which have a level of about $1 \%$ to $2 \%$ of the continuum and vanish in the difference between two consecutive states of polarization, and polarization fringes (due to the variable retarders), which are more than 100 times fainter $(0.01 \%$ of the continuum) and which appear only after a long time of integration in the difference between alternate polarizations. Such fringes are hard to treat, because the residual pattern generally shifts slowly during the observing run and is comparable to weak solar polarizations signals $\left(10^{-4}\right)$. Flat fielding is very useful to precisely determine the transmission of the instrument for the two states of polarization (a difference of about $0.2 \%$ is typical, depending on the line, because the transmission of the NLC varies slightly with retardance). We make the assumption that the polarization rate is null at disk center in the quiet sun, and determine the zero level of the

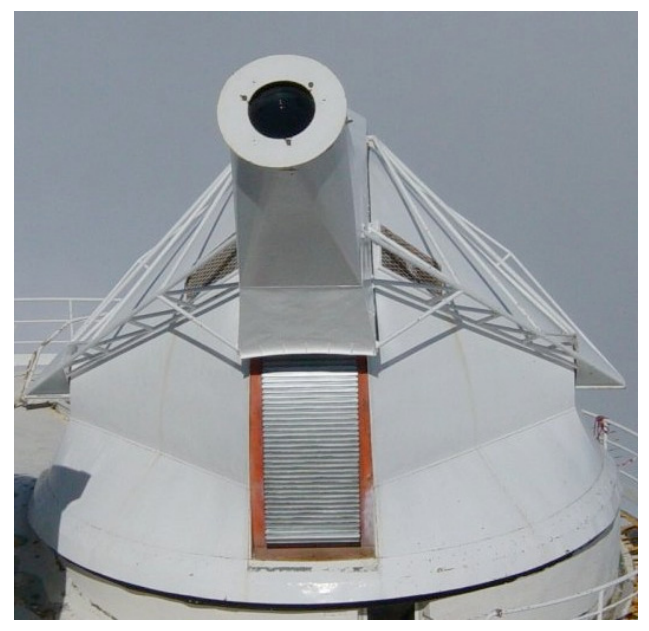

Fig. A.5. Mehltretter's ring to avoid heating of the lens holder.

polarization signal from the flat field as a function of wavelength (the retardance in the observed spectral range varies faintly with wavelength and consequently induces a $Q / I$ slope of about $5 \times$ $10^{-4}$ per nm which is easily corrected by a linear adjustment).

\section{A.6. Stability of polarization measurements}

The stability of polarization measurements may be affected by several effects, such as solar, but also seeing and instrumental variations. It was noticed in the past that the image quality deteriorates when the main objective holder is heated by the sun. For that reason, P. Mehltretter suggested to protect the holder with a reflective ring with clear aperture corresponding exactly to the diameter of the lens (Fig. A.5). We discovered that instrumental depolarization (up to $-25 \%$ ) occurs without the ring two hours before and after the meridian. With the ring, we measured only small fluctuations which are mainly due to variations of the limb distance which can move within a few arcsec between each test, as we do not have a precise guiding system. In conclusion, if some instrumental depolarization remains, it is not easily detectable.

\section{Appendix B: The experiment setup in imagery mode}

The instrumental setup is presented in Fig. B.1. The polarimetry system is identical to the one used in spectroscopy, but the magnification is smaller $(\times 2)$, so that we work at $f / 30$ instead of $f / 130$ in spectro polarimetry. The field of view is $100^{\prime \prime} \times 120^{\prime \prime}$ with a pixel size of $0.1^{\prime \prime}$. The detector is installed on the optical rail, at the location of the injection mirror to the spectrograph which is removed for the circumstance.

\section{Appendix C: Ongoing developments for the spectroscopic mode}

From a technical point of view, we know that the present version of our polarimeter has still some limitations which will be removed in the future. In particular, polarization signals $I \pm P(P=Q, U, V)$ are observed sequentially because the analyser is a simple (high quality) dichroic linear polarizer. A new device is under development to replace it (Fig. C.1), consisting of a birefringent linear polarizing beam splitter shifter which will allow one to observe simultaneously both 
J.-M. Malherbe et al.: Observations of the second solar spectrum, Online Material p 5

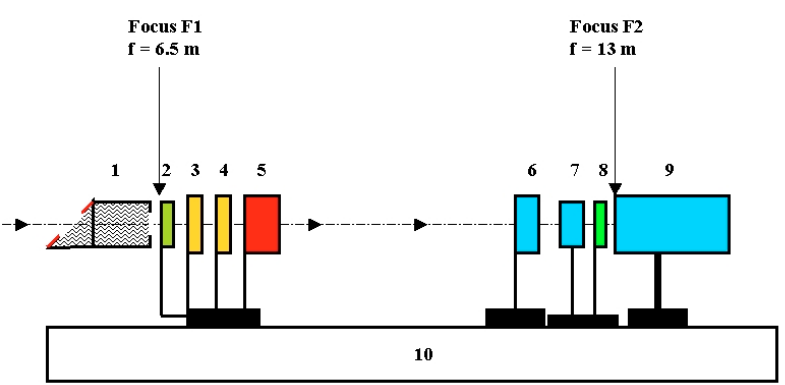

1: water cooling device (field stop) 2: UV/IR filter $380-700 \mathrm{~nm}$

3: variable retarder

4: variable retarder 2

5: magnification lens $(\mathrm{f}=95 \mathrm{~mm})$

Fig. B.1. The optical setup between the primary focus F1 and the secondary focus $\mathrm{F} 2$ in imagery mode (the $50 \mathrm{~cm}$ refractor is located on the optical axis at left).

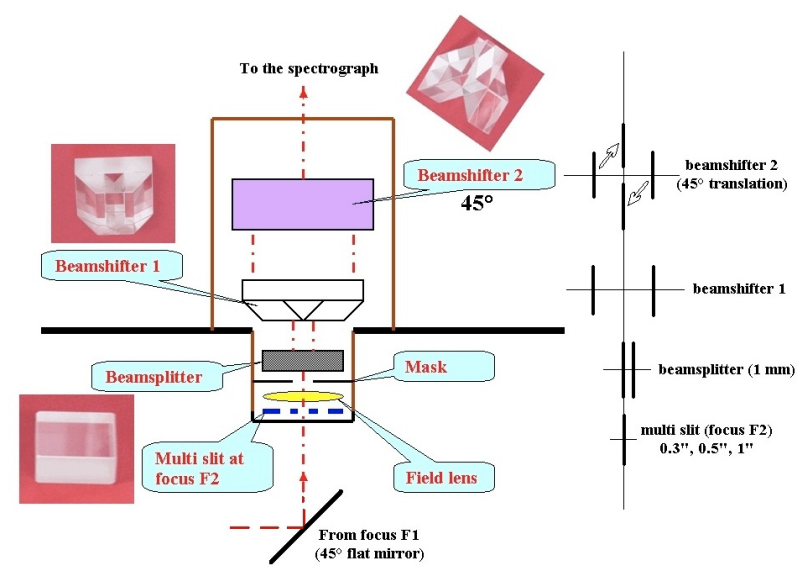

Fig. C.1. Details of the polarizing beam splitter shifter (end 2006).

signals, so that $I \pm P$ will be focused at the same time on the CCD detector. As a consequence, the field of view will be reduced by a factor of two, from $140^{\prime \prime}$ to $70^{\prime \prime}$ along the sun. The beam splitter shifter will be tested by the end of

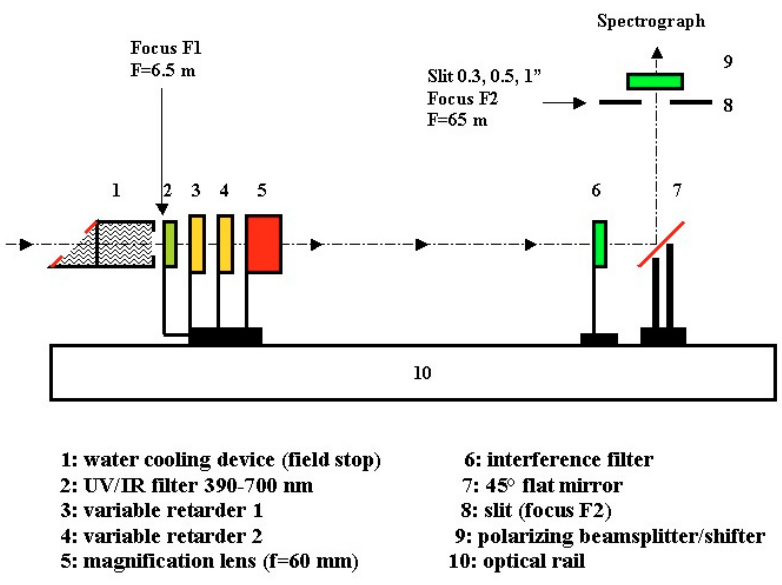

Fig. C.2. The experiment setup in spectroscopic mode with the polarizing beam splitter shifter (end 2006).

2006 and will be located at focus F2 (Fig. C.2) just after the entrance slit of the spectrograph. This improvement is of special importance for the polarimetry of specific solar structures observed on the disk (active regions, flux tubes) or at the limb (spicules, prominences) which require perfect coalignment of both signals, in order to eliminate seeing induced polarimetric cross talk. But observations of the second solar spectrum do not suffer from this default, because of statistical effects due to the large number (thousands) of spectra required to achieve a convenient signal to noise ratio. Beam exchange will be available with the new beam splitter, especially for the measurement of weak polarization signals. 